\title{
A Programming Model and Foundation for Lineage-Based Distributed Computation
}

\author{
PHILIPP HALLER \\ KTH Royal Institute of Technology, Sweden \\ HEATHER MILLER \\ EPFL, Switzerland and Northeastern University, USA \\ NORMEN MÜLLER \\ BearingPoint Software Solutions GmbH, Germany
}

\begin{abstract}
The most successful systems for "big data" processing have all adopted functional APIs. We present a new programming model we call function passing designed to provide a more principled substrate, or middleware, upon which to build data-centric distributed systems like Spark. A key idea is to build up a persistent functional data structure representing transformations on distributed immutable data by passing well-typed serializable functions over the wire and applying them to this distributed data. Thus, the function passing model can be thought of as a persistent functional data structure that is distributed, where transformations performed on distributed data are stored in its nodes rather than the distributed data itself. One advantage of this model is that failure recovery is simplified by design-data can be recovered by replaying function applications atop immutable data loaded from stable storage. Deferred evaluation is also central to our model; by incorporating deferred evaluation into our design only at the point of initiating network communication, the function passing model remains easy to reason about while remaining efficient in time and memory. Moreover, we provide a complete formalization of the programming model in order to study the foundations of lineage-based distributed computation. In particular, we develop a theory of safe, mobile lineages based on a subject reduction theorem for a typed core language. Furthermore, we formalize a progress theorem which guarantees the finite materialization of remote, lineage-based data. Thus, the formal model may serve as a basis for further developments of the theory of data-centric distributed programming, including aspects such as fault tolerance. We provide an open-source implementation of our model in and for the Scala programming language, along with a case study of several example frameworks and end-user programs written atop of this model.
\end{abstract}

\section{Contents}

1 Introduction $\quad 2$

1.1 Contributions 3

2 Overview $\quad 4$

2.1 Essence 4

2.2 Basic Usage 5

2.3 Programming Model 6

2.4 Primitives $\quad 8$ 
$\begin{array}{lll}2.5 & \text { Limitations } & 14\end{array}$

3 Examples 14

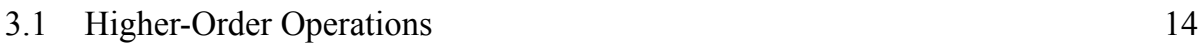

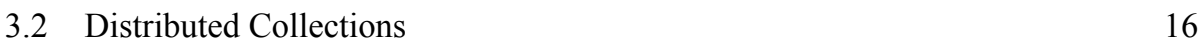

$\begin{array}{lll}3.3 & \text { K-Means Clustering } & 17\end{array}$

4 Formalization 18

$\begin{array}{ll}4.1 \text { Operational Semantics } & 19\end{array}$

4.2 Type Assignment 23

4.3 Well-Formed Configurations $\quad 25$

4.4 Persist and Unpersist $\quad 25$

5 Correctness Properties 28

5.1 Subject Reduction $\quad 28$

$\begin{array}{lll}5.2 \text { Progress } & 28\end{array}$

6 Related Work 30

7 Future Work and Conclusion 32

7.1 Ongoing and Future Work $\quad 32$

$\begin{array}{lll}7.2 \text { Conclusion } & 34\end{array}$

A Illustrated Listings $\quad 36$

A.1 Matching Vehicles and Owners $\quad 36$

A.2 K-Means Clustering $\quad 36$

$\begin{array}{ll}\text { B Proofs } & 37\end{array}$

$\begin{array}{lll}\text { B.1 Proof of Theorem } 4.1 & 37\end{array}$

B.2 Proof of Theorem 5.2 38

B.3 Proof of Theorem 5.5 44

References $\quad 45$

\section{Introduction}

Data-centric programming is growing in importance with the most successful systems for programming with "big data" all adopting ideas from functional programming; i.e., programming with first-class functions. These functional ideas are often touted to be the key to the success of these frameworks. Functional, declarative interfaces to data, distributed over tens to thousands of nodes, provide a more natural way for end-users and data scientists to reason about data.

While leveraging functional programming concepts, popular implementations of Google's MapReduce (Dean \& Ghemawat, 2008) model, such as Apache Hadoop's MapReduce Framework (Apache, 2015) for Java, have been developed without making use of functional language features such as closures. For nearly a decade, the Apache Hadoop open source interpretation of this model grew in popularity, remaining largely unchallengedcausing nearly all of industry to synchronize on this one implementation for most all largescale data processing needs.

However, in recent years, a new generation of distributed systems for large-scale data processing have suddenly cropped up, built on top of emerging functional languages like Scala (Odersky et al., 2010); such systems include Apache Spark (Zaharia et al., 2010), 
Twitter's Scalding (Twitter, 2015), and Scoobi (NICTA, 2015). These systems make use of functional language features in Scala in order to provide high-level, declarative APIs to end-users. Further, the benefits provided by functional programming have also won over framework designers as well-some have noticed that immutability, and data transformation via higher-order functions makes it much easier, by design, to tackle concerns central to distributed systems such as concurrency.

While widely adopted in practice, the aforementioned programming systems are not without important issues. On the one hand, their programming interfaces do not prevent common usage errors, such as unsafe closure serialization. As a result, the complexities of distribution may trickle even to end users, who are increasingly non-expert users. On the other hand, the foundations of their programming models remain largely unclear, in particular, foundations of core aspects such as fault tolerance, a critical aspect for distributed operation on a large scale.

This paper introduces a new programming model which embraces the principle of stationary data containers and mobile functions ("move computation to the data"). It can be viewed as a generalization of the MapReduce/Spark programming model. Our programming model which we call function passing can be thought of as a programming model for a middleware, meant to underly systems like Spark. Function passing adopts the concept of lineage which is used by systems like Spark to handle fault tolerance. Importantly, lineage-based fault tolerance is facilitated by the core computational principle of functional transformations on immutable data.

The programming model is based on functional abstractions for lineage-based distributed computation. In order to prevent common usage errors, the model builds upon two previous veins of work-type-safe serialization based on functional pickler combinators (Kennedy, 2004; Elsman, 2005; Miller et al., 2013; Rossberg et al., 2004), and serializable closures (Epstein et al., 2011; Miller et al., 2014). We believe this unique combination of functional programming techniques provides a more principled substrate upon which to build datacentric, distributed systems.

Importantly, we provide a complete formalization of the programming model. In particular, we develop a theory of safe, mobile lineages based on a subject reduction theorem for a typed core language. Furthermore, we formalize a progress theorem which guarantees the finite materialization of remote, lineage-based data. To our knowledge, these theorems constitute the first correctness results for a programming model for lineage-based distributed computation. Thus, our formal model may serve as a basis for further developments of the theory of data-centric distributed programming, including aspects such as fault tolerance.

\subsection{Contributions}

This paper makes the following contributions:

- A new data-centric programming model for functional processing of distributed data which provides abstractions for building fault-tolerant distributed systems, including first-class lineages. The main computational principle is based on the idea of sending safe, guaranteed serializable functions to stationary data containers. Using standard monadic operations, our model enables creating directed acyclic graphs (DAG) of 
computations. Deferred evaluation enables optimizations such as operation fusion while keeping programs simple to reason about.

- A formalization of lineage-based distributed computation based on a small-step operational semantics. Our formalization extends previous theories of serializable closures to serializable lineages. The technical development enabling this extension combines (a) serializable types, (b) "static" closures, and (c) lineages.

- A proof of a subject reduction theorem for a typed, distributed core language based on lineages. To our knowledge we present the first such proof for a lineage-based distributed programming model.

- A proof establishing the preservation of lineage mobility by reduction for a typed, distributed core language. This property provides a foundation for lineage-based fault tolerance.

- The formalization of a progress theorem, guaranteeing the finite materialization of remote, lineage-based data, including a detailed proof sketch.

- A distributed implementation of the programming model in and for Scala as a middleware. ${ }^{1}$ In addition, we present prototype versions of programming abstractions provided by popular frameworks like Apache Spark and MBrace using the function passing model, and end-user applications we have built using these prototypes.

In the rest of the paper, our approach is as follows. First, we describe our model on a high level, elaborating upon key benefits and trade-offs, and then we zoom in to make each component part of our model more precise. We describe the basic model this way in Section 2. In Section 3 we go on to show how essential higher-order operations on distributed frameworks like Apache Spark can be implemented in terms of the primitives presented in Section 2. We formalize our programming model in Section 4, providing an operational semantics and a type system. In Section 5 we present the proof of a subject reduction theorem and formalize important progress properties. Finally, we discuss related work in Section 6, and conclude in Section 7.

\section{Overview}

\subsection{Essence}

The function passing model is intended to act as a middleware upon which to build up data-centric distributed systems like Spark.

In the broadest sense, it can be thought of as a sort of persistent functional data structure with monadic operations and structural sharing. However, rather than containing pure data, instead this data structure represents a graph ${ }^{2}$ of functional transformations, or operations, on distributed data. The root node contains immutable data read from stable storage (e.g., Amazon S3); edges represent functional transformations. Said another way, the core of the function passing model can be thought of as a persistent functional data structure representing a history of the operations performed on some data, rather than the data itself.

1 See the Git repository at https://github.com/heathermiller/f-p/, branch jfp.

2 a directed acyclic graph (DAG) 
Importantly, since this DAG of computations is a persistent data structure itself, it is safe to exchange (copies of) subgraphs of a DAG between remote nodes. Subgraphs of the DAG are called lineages; lineages enable restoring the data of failed nodes through re-applying their transformations. This sequence of applications must begin with data available from stable storage.

Central to the function passing model is the careful use of deferred evaluation. Computations on distributed data are typically not executed eagerly; instead, applying a function to distributed data just creates an immutable, local lineage. To make a network call and thus obtain the result of a computation, it is necessary to first "kick off" the computation in order to materialize the nodes of its lineage. Within our programming model, this force operation $^{3}$ makes network communication (and thus possibilities for latency) explicit, which is considered to be a strength when designing distributed systems (Waldo et al., 1996). Deferred evaluation also enables optimizing distributed computations through operation fusion, which avoids the creation of unnecessary intermediate data structures-this is efficient in time as well as space. This kind of optimization is particularly important and effective in distributed systems (Chambers et al., 2010). For these reasons, we believe that deferred evaluation should be viewed as an enabler in the design of distributed systems.

\subsection{Basic Usage}

We begin with a simple visual example to illustrate the intuition behind the function passing model. The function passing model consists of three main components:

- Silos, stationary, typed, immutable data containers.

- Silo references to local or remote silos.

- Spores, safe, serializable functions.

The main handle users have to the framework is via SiloRefs. A SiloRef[T] can be thought of as an immutable handle to a remote value of type T contained within a corresponding silo. Users interact with this remote data by applying functions (as spores) to silo references. Those functions are transmitted over the wire and later applied to the data within the corresponding silo. As is the case for persistent data structures, when a function is applied to a piece of remote data via a SiloRef[T], a new SiloRef [ $T$ ' $]$, representing a new silo containing the transformed data $T^{\prime}$, is returned.

We go into more detail about each of these components later in Section 2.3.

The simplest illustration of the model is shown in Figure 1 (time flows vertically from top to bottom). Here, we start with a SiloRef[T] which points to a piece of remote data contained within a Silo[T]. When the function, shown as $\lambda$, of type $T \Rightarrow \operatorname{SiloRef}[S]$ is applied to SiloRef[T] and "forced" (sent over the network), a new silo reference of type SiloRef[S] is immediately returned. Note that SiloRef[S] contains a reference to its parent silo reference, SiloRef [T]. (This is how lineages are constructed.) Meanwhile, the function is asynchronously sent over the network and is then applied to Silo[T], eventually producing a new Silo[s] ${ }^{4}$ containing the data transformed by function $\lambda$. This new SiloRef[S]

3 called send(), discussed in more depth in Section 2.4 along with the other primitive operations in the function passing model.

4 New silos are materialized on the same node as their parent. 
can be operated on even before its corresponding silo is materialized (i.e., before the data in Silo[S] is computed) - the function passing framework queues up operations applied to SiloRef[S] and applies them when Silo[S] is fully materialized.

Different sorts of complex DAGs can be asynchronously built up in this way. Though first, to see how this is possible, we need to develop a clearer idea of the primitive operations available on silo references and their semantics. We describe these in the following.

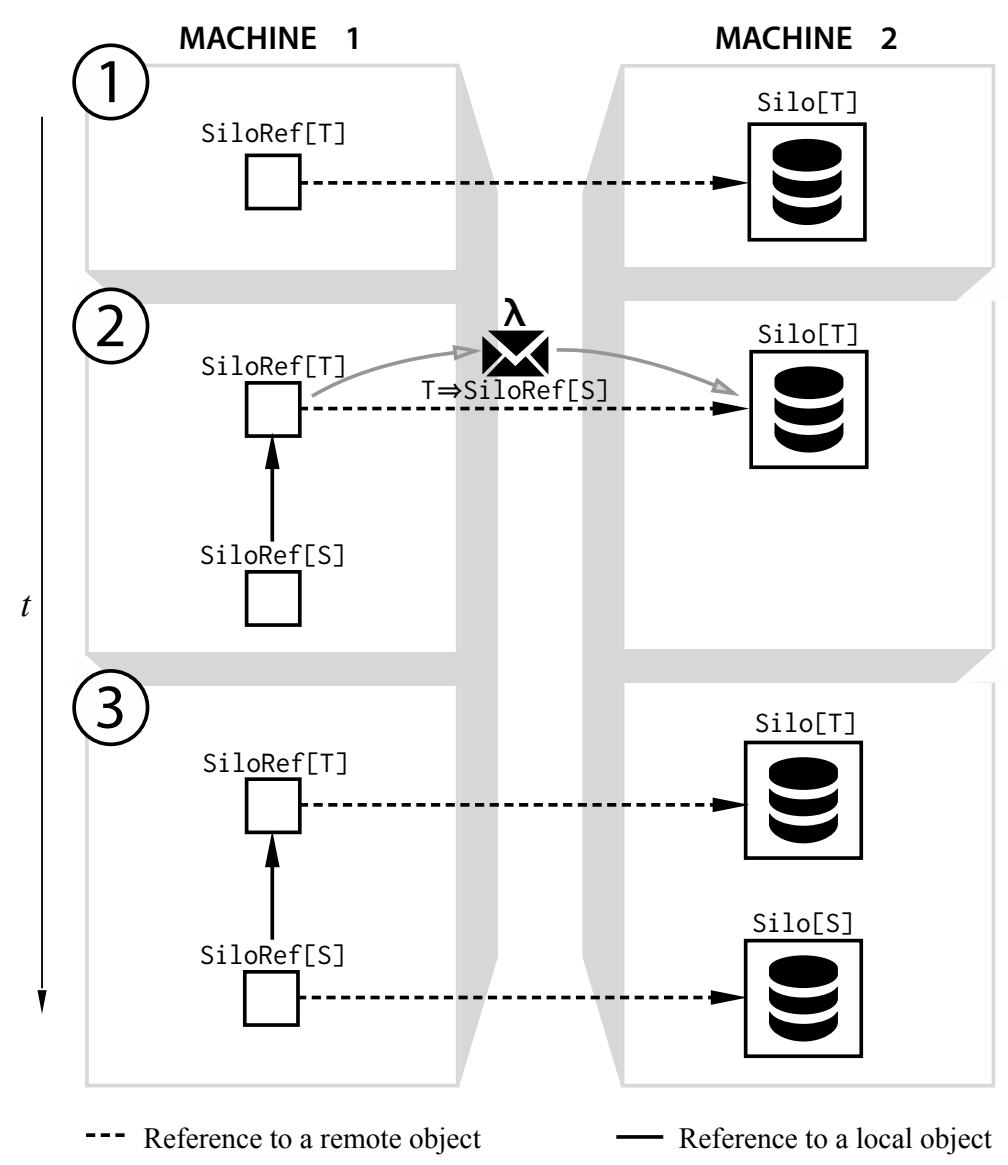

Fig. 1. Basic function passing model.

\subsection{Programming Model}

With a basic intuition under our belt for how distributed computation is performed in the function passing model, we focus now on its three main components; silos, silo references, and spores.

Silos. A silo is a typed and immutable data container. The container is stationary in the sense that it does not move between machines - it remains on the machine where it was created. Data stored in a silo may either be loaded from stable storage, such as a distributed 
file system, or it may be the result of a computation. Thus, the data stored in a silo is often not stationary (in contrast to the containers, the silos, which are stationary), because it may need to be transferred to other machines to compute the contents of other silos. A program operating on data stored in a silo can only do so using a reference to the silo.

Silo references. Similar to a proxy object, a silo reference represents, and allows interacting with both local and remote silos. Silo references are immutable, storing identifiers to locate possibly remote silos. They are also typed (SiloRef[T]) corresponding to the type T of their silo's data, leading to well-typed network communication. A silo reference provides two principle operations: apply and send. The apply method makes use of deferred evaluation; it eventually applies a user-defined function to data pointed to by the SiloRef[T], creating a new silo containing the result of this application, though this application is deferred. That is, this computation is only "kicked off" when the send method is invoked. This makes it possible to queue up transformations in order to optimize network communication. Note that the user-defined function passed to apply returns a SiloRef[S] whose contents is transferred to the new silo returned by apply. Essentially, apply enables accessing the contents of (local or remote) silos from within remote computations. We illustrate these primitives in more detail in Section 2.4.

Spores. Spores (Miller et al., 2014) are safe closures that are guaranteed to be serializable and thus distributable. Essentially, a spore is a closure-like abstraction with associated type rules which gives authors of distributed frameworks a principled way of controlling the environment which a closure (provided by client code) can capture. This is achieved by (a) enforcing a specific syntactic shape which dictates how the environment of a spore is declared, and (b) providing additional type-checking to ensure that types being captured have certain properties.

A spore consists of two parts: the spore header, composed of a list of value definitions, and the spore body, a regular closure; sometimes referred to as the "spore closure." This shape is illustrated in Figure 2.

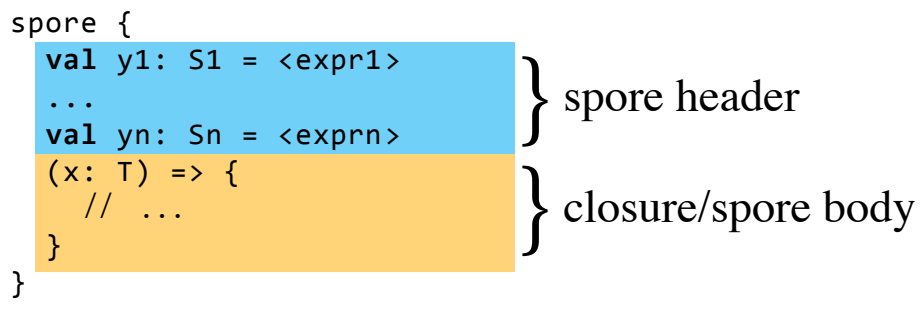

Fig. 2. The shape of a spore.

The characteristic property of a spore is that the spore body is only allowed to access its parameter, the values in the spore header, as well as top-level singleton objects (Scala's form of modules). The spore closure is not allowed to capture variables other than those declared in the spore header (i.e., a spore may not capture variables in the environment). By enforcing this shape, the environment of a spore is always declared explicitly in the spore 


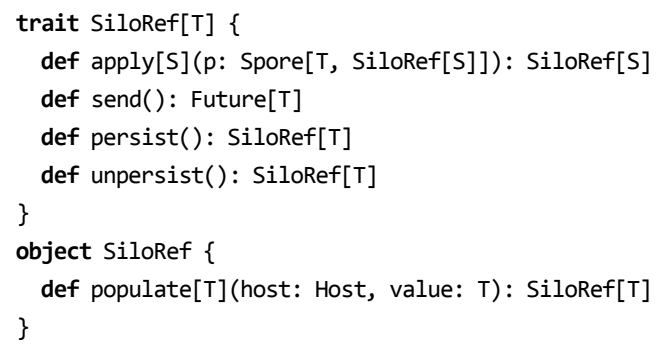

Fig. 3. Type signatures of primitive operations.

header, which avoids accidentally capturing problematic references. Moreover, importantly for object-oriented languages like Scala, it is no longer possible to accidentally capture the this reference.

Spores also come with additional type-checking. Type information corresponding to captured variables are included in the type of a spore. This enables authors of distributed frameworks to customize type-checking of spores to, for example, exclude a certain type from being captured by user-provided spores. Authors of distributed frameworks may kick on this type-checking by simply including information about excluded types (or other typebased properties) in the signature of a method. A concrete example would be to ensure that the map method on RDDs in Apache Spark (a distributed collection) accepts only spores which do not capture sparkContext (a non-serializable internal framework class).

\subsection{Primitives}

There are five basic primitive operations on silo references that together can be used to build the higher-order operations common to popular data-centric distributed systems. (How to build some of these higher-order operations is described in Section 3.). In this section we introduce these primitives in the context of a running example. These primitives include apply, send, persist, unpersist, and populate; their type signatures are shown in Figure 3.5 Note that populate does not operate on a SiloRef, unlike the other primitives. Instead, populate is used to create new SiloRefs; therefore, it is defined as a (factory) method in the SiloRef singleton object. ${ }^{6}$

apply. def apply[S](p: Spore[T, SiloRef[S]]): SiloRef[S]

The apply method takes a spore that is to be applied to the data in the silo associated with the given SiloRef (i.e., the receiver of the method call). Rather than immediately sending the spore across the network, and waiting for the operation to finish, the apply method's evaluation is deferred. Without involving any network communication, it immediately returns a SiloRef referring to a new, to-be-created silo. This new silo reference only contains

5 A trait in Scala can be thought of as an abstract class supporting mixin composition, essentially providing a safe form of multiple inheritance (Odersky \& Zenger, 2005).

6 Singleton objects are Scala's form of modules. The name of a singleton object refers to a value. Since in Scala the namespace for types is disjoint from the namespace for values, the trait and the singleton object can have the same name. 
lineage information, namely, a reference to the original siloRef and a reference to the argument spore. As we explain below, another method, send, must be called explicitly to force the materialization of the result silo. Note that the result type of the spore parameter is a SiloRef[S]. Semantically, the new silo created by apply is defined to contain the data of the silo that the user-defined spore returns. This way, the apply combinator enables expressing computation DAGs.

To better understand how DAGs are created and how remote silos are materialized, we will develop a running example throughout this section. Given a silo containing a list of Person records, the following invocation of apply defines a (not-yet-materialized) silo containing only the records of adults (graphically shown in Figure 5, part 1):

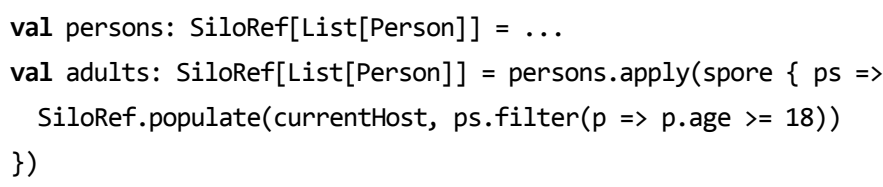

When the spore is received on the machine hosting the silo corresponding to the persons silo reference, it is applied to the contents of the silo, a List[Person]. The body of the spore (a) filters this list (ps), creating a new list of adults, and (b) populates a new silo with the result of the filter invocation. Note that populate takes the host of the new silo as an argument; in the above example, this argument is currentHost which returns the host currently computing the silo's contents.

Section 2.4.1 below describes additional ways to create new silos. However, in each case, the host on which the silo should be created must be specified. The reason is that each instance of SiloRef $[\mathrm{T}]$ contains the host of the corresponding silo; this information is necessary for accessing the silo's data. Given the fact that the host of a SiloRef is fixed, silorefs are not suitable for direct use by applications requiring fault tolerance. Instead, siloRefs should be regarded as a (low-level) building block for distributed systems which implement fault-recovery mechanisms on top of the basic functionality provided by siloRefs.

The apply combinator enables expressing also more interesting computation DAGs. For example, consider the problem of combining the information contained in two different silos (potentially located on different hosts). Suppose the information of a silo containing vehicle records should be enriched with other details only found in the adults silo. In the example shown in Figure 4 apply is used to create a silo of (Person, vehicle) pairs where the names of person and vehicle owner match.

Here, it is necessary to read the data of the vehicles silo in addition to the persons, the list of Person records. This requires calling apply on localvehicles on line 6, whose argument spore captures the persons list and takes the vs list as a parameter; thereby, the two lists can be combined, and the result stored in a new silo (line 9). Note that with the use of apply on line 3, the call to localvehicles.apply (..) on line 6 creates the final result silo, whose data is then also contained in the owners silo declared on line 2. (See Appendix A.1 for a diagram illustrating also the use of regular Scala collection combinators in the listing.)

To illustrate the data flow between hosts, let us kick off the materialization of the involved silos:

val adults: SiloRef[List[Person] $]=\ldots$

val vehicles: SiloRef[List $[$ Vehicle $]]=\ldots$ 


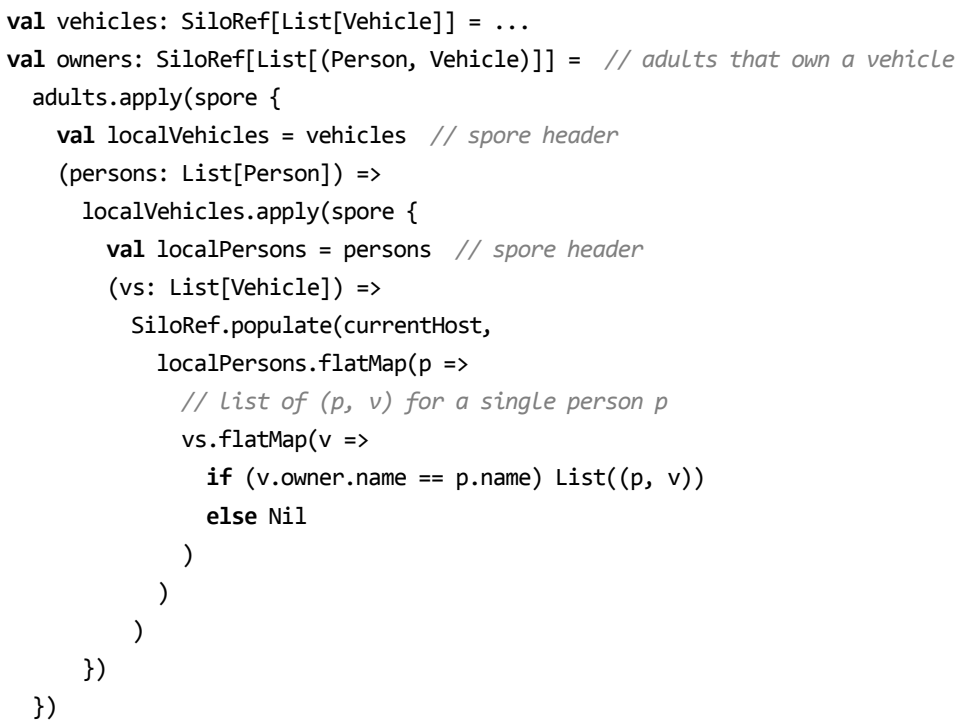

Fig. 4. Matching persons and vehicle owners using the apply combinator.

val owners: Future[List $[($ Person, Vehicle $)]]=$

adults.apply(...).send()

For illustration we use the informal notation @m to denote the location of a value. We assume that the silo references are at machine $\mathrm{m} 1$ but the actual data is distributed over $\mathrm{m} 2$ and $\mathrm{m} 3$ (note that these locations are different from Figure 5, part 2; however, they help make the required data transfers more precise):

adults@ @1 --> Silo[List[Person]] @m2

vehicles@m1 -->Silo[List[Vehicle]] @ m3

To create owners, we must combine data hosted at $\mathrm{m} 2 \mathrm{with}$ data hosted at $\mathrm{m} 3$. First, adults. apply transfers its spore, i.e., the silo reference vehicles and the closure

persons $=>\ldots$, to $\mathrm{m} 2$ hosting the referenced silo of grown-up Person records. Next, localvehicles. apply transfers its spore, i.e., the collection of adults persons and the closure vehicles $\Rightarrow$..., to $m 3$ hosting the referenced silo of vehicle records. Now, at $m 3$, we have all required information: the adults persons, the vehicles vehicles, and the necessary computations to combine corresponding records, resulting in a new (anonymous) silo reference at $\mathrm{m} 2$ referencing the new silo of (Person, Vehicle) records hosted at $\mathrm{m} 3$. This new silo reference at $\mathrm{m} 2$ is used to transfer its referenced data from $m 3$ to $m 2$, the origin of the apply, and, eventually, to $\mathrm{m} 1$ where the materialization has been kicked off.

To reduce the amount of data that is transferred, the implementation in fact leverages silo reference proxies, delegating to the actual data:

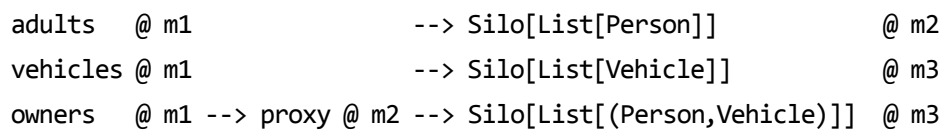



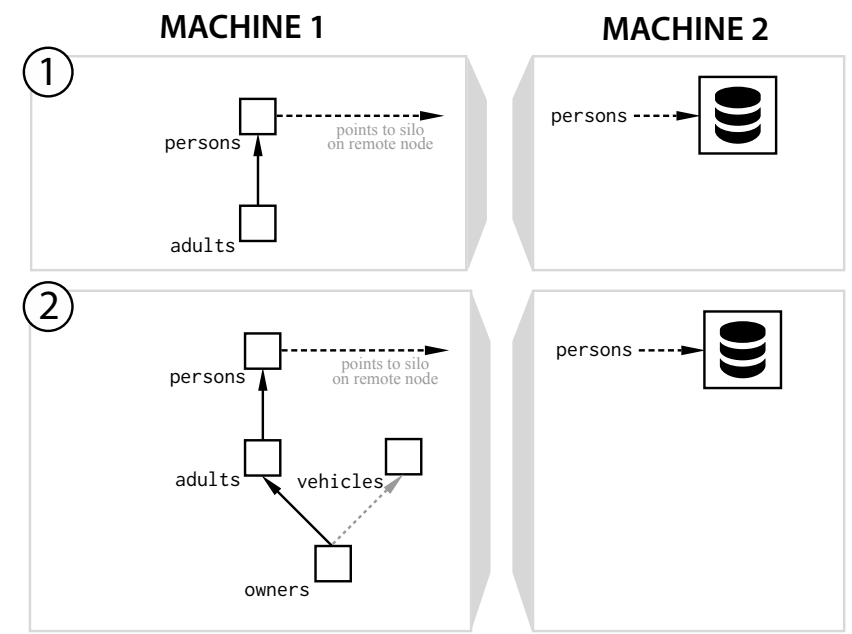

(3)
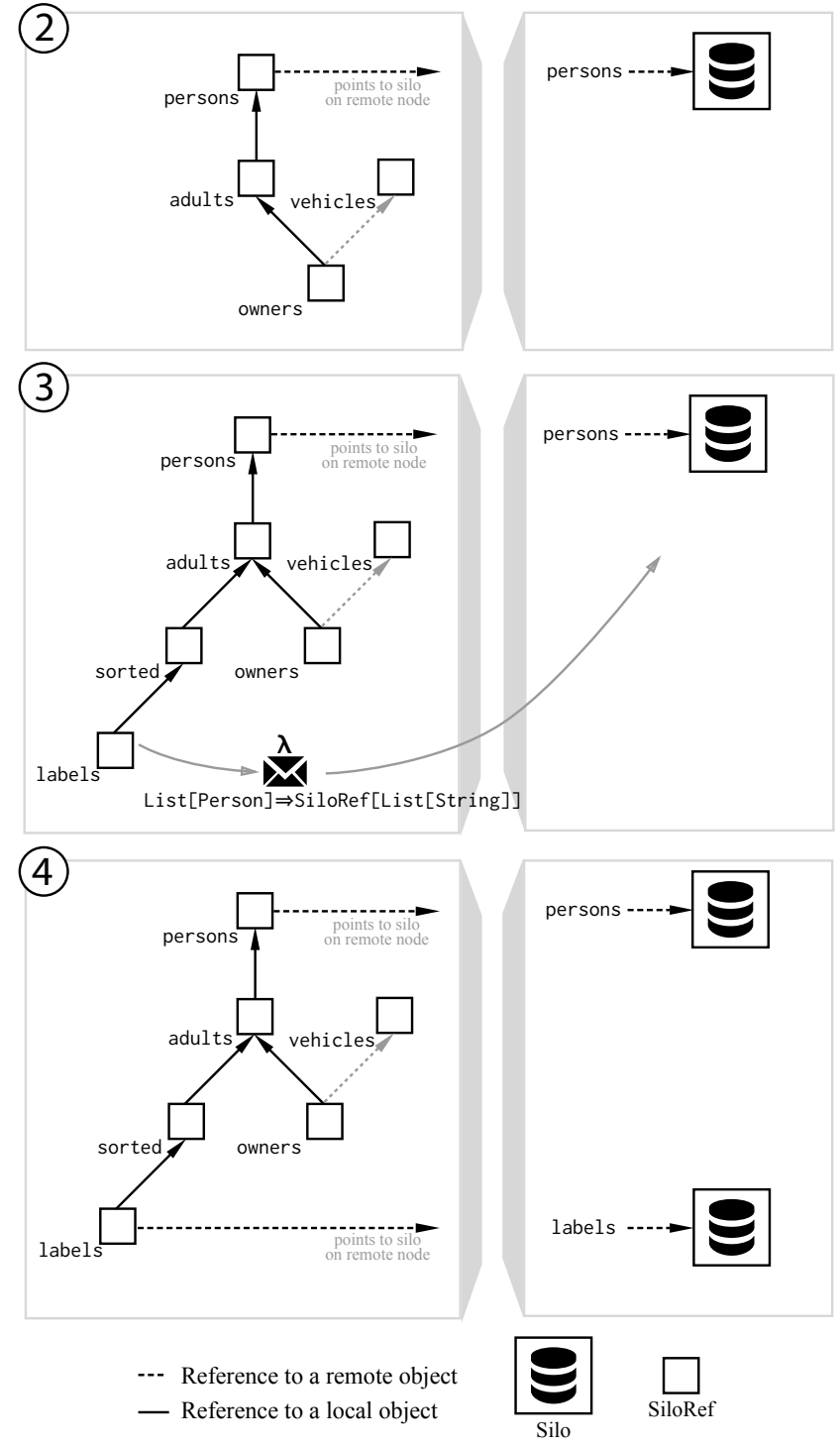

Fig. 5. A simple DAG in the function passing model.

The anonymous silo reference created at $\mathrm{m} 2$ will not lead to a materialization of its contents at $\mathrm{m} 2$; instead, the silo reference functions as a proxy for the data hosted at $\mathrm{m} 3$. As a result, when $\mathrm{m} 1$ requests the actual list of persons owning a vehicle, it is retrieved from $\mathrm{m} 3$. Avoiding a materialization also on $\mathrm{m} 2$ alleviates network communication overhead. 
Finally, note that the spore passed to apply on line 3 in Figure 4 declares the capturing of the vehicles silo reference in its spore header. The spore header spans all variable definitions between the spore marker and the parameter list of the spore's closure. The spore header defines the variables that the spore's closure is allowed to access. Essentially, spores limit the free variables of their closure's body to the closure's parameters and the variables declared in the spore's header.

send. def send(): Future[T]

As mentioned earlier, the execution of computations built using silo references is deferred. The send operation forces the deferred computation defined by the given SiloRef [ $\mathrm{T}]$. Forcing is explicit in our model, because it requires sending the lineage to the remote node on which the result silo should be created. Given that network communication has a latency several orders of magnitude greater than accessing a word in main memory, providing an explicit send operation is a judicious choice (Waldo et al., 1996).

To enable materialization of remote silos to proceed concurrently, the send operation immediately returns a future (Haller et al., 2012). This future is then asynchronously completed with the data of the given silo. Since calling send will materialize a silo on the same node as its parent, and will send its resulting data to the current node, send should only be called on silos with reasonably small data (for example, in the implementation of an aggregate operation such as reduce on a distributed collection).

persist. def persist(): SiloRef[T]

The performance of typical data analytics jobs can be increased dramatically by caching large datasets in memory (Zaharia et al., 2010). To do this, silos containing computed datasets need to be materialized, and furthermore, re-materialization should be avoided when datasets are used multiple times.

The only way, that we have shown so far, to materialize a silo is using the send primitive. However, using send does not prevent the system from evicting the silo from memory at a later point in time, e.g., when its host is running low on memory. In addition, send transfers the contents of the silo to the requesting host-too much if a large remote dataset should merely be cached in memory remotely.

Therefore, we provide an additional primitive called persist which immediately returns a SiloRef representing a silo which (a) has the same data when materialized, and (b) is guaranteed to remain in memory as long as at least one host has persisted the siloRef.

Given the running example so far, we can add another lineage branching off of adults by sorting the list of Person records by age, and producing a greeting string for each record:

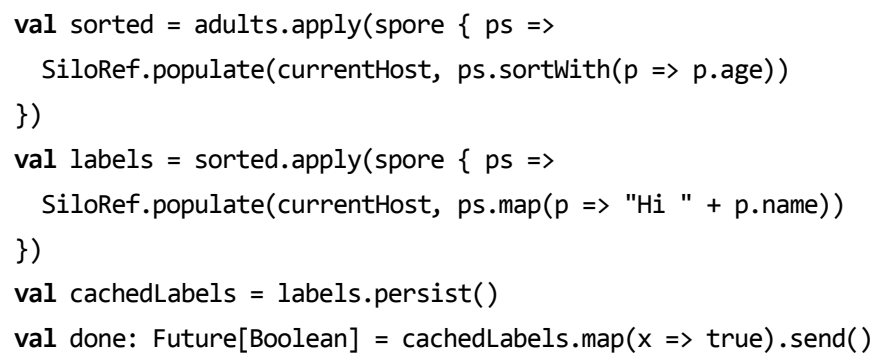


The cachedLabels silo contains the same (logical) data as the labels silo, however the data of cachedLabels is cached in memory. Thus, cachedLabels needs to be materialized just once. In order to "kick off" remote computation, it is still necessary to call send; however, it is always possible to "prefetch" data into remote memory by mapping the data to a trivial value (true above) and invoking send to materialize the lineage. ${ }^{7}$ The lineage for the above example looks as illustrated in Figure 5.

unpersist. def unpersist(): SiloRef[T]

The final primitive operation on silo references is unpersist. By invoking unpersist on a silo reference $r$ the current host declares that it is no longer interested in the data of $r$. As a result, unless other hosts have persisted $r$, the memory occupied by the data of $r$ may be reclaimed without negatively impacting performance. Just like persist, invoking unpersist immediately returns a SiloRef representing a silo which may not be cached in memory. However, if another host has persisted $r$, the silo's data remains cached in memory. In the context of future work we plan to investigate techniques for inferring invocations of unpersist in order to automatically and efficiently manage silo memory (see Section 7).

\subsubsection{Creating Silos}

As mentioned earlier, besides a type definition for SiloRef $[\mathrm{T}]$, our framework also provides a companion singleton object. The singleton object provides a variety of factory methods for obtaining silo references referring to silos populated with some initial data: ${ }^{8}$

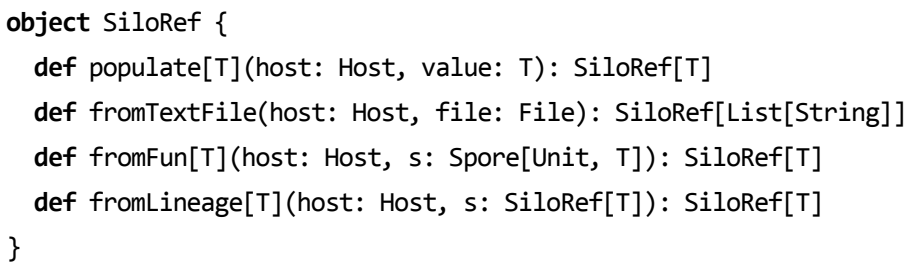

Each of the factory methods has a host parameter that specifies the target host (address/port) on which to create the silo. Note that the fromfun method takes a spore closure as an argument to make sure it can be serialized and sent to host. In each case, the returned SiloRef[T] contains its host as well as a host-unique identifier. The fromLineage method is particularly interesting as it creates a copy of a previously existing silo based on the lineage of a silo reference s. Note that only the silo reference is necessary for this operation to successfully complete; the silo is not required to be materialized.

\subsubsection{Type Polymorphism and Silos/SiloRefs}

An important property of silos is that they are polymorphic in the type of data that they hold (Silo[T]). Importantly, silos may not only store collections; silos are polymorphic in

7 Our system also provides a cache operation which abbreviates this pattern: semantically, ref.cache( $)$ is equivalent to ref.persist ()$\cdot \operatorname{map}(x \Rightarrow()) \cdot \operatorname{send}()$.

8 For clarity, only method signatures are shown. 
the type of their entire dataset. For example, a silo might contain a Red-Black tree with elements of type Person for some ADT Person, ordered by one of the fields of the Person type. Another silo might contain a completely different collection type, say, a linked list. This type polymorphism enables optimizing silos according to their data access patterns. Given that different data types may have specialized operations (e.g., a tree map could provide a range projection), the key to enabling this type polymorphism is the fact that a spore, sent to a silo, may apply arbitrary functions to the silo's data. Thus, the SiloRef API itself is not limited to providing just a fixed set of built-in operations (in contrast to RDDs in Apache Spark, for example).

\subsection{Limitations}

Although fairly small and simple, the introduced programming model is quite flexible, and we have used it to implement a variety of examples and abstractions from the literature (see Section 3). However, as presented, the function passing model also has important limitations. Two principle limitations pertain to distributed data and distributed behavior:

- Data maintained in silos is immutable. As a result, computational patterns based on mutating distributed state cannot be expressed in the model. An example would be (potentially long-lived) distributed graph data that is asynchronously updated. Another example would be mutable, distributed data structures such as CRDTs (Shapiro et al., 2011).

- A computation cannot spawn independent activities. While it is possible to create spore closures and send them to remote hosts for execution, a spore is applied only once, to materialize (the contents of) a silo. However, there is no way to create a behavior which remains active in some way across multiple interactions, like actors (Agha, 1986) or processes in the $\pi$-calculus (Milner et al., 1992).

\section{Examples}

The introduced primitives enable expressing surprisingly intricate computational patterns.

Higher-order operations such as variants of map, reduce, and join, operating on collections of data partitions, distributed across a set of hosts, are required when implementing abstractions like Spark's distributed collections (Zaharia et al., 2010). Section 3.1 demonstrates the implementation of some such operations in terms of silos.

Section 3.2 shows an extension of the higher-order operations of Section 3.1, providing a distributed collections abstraction reminiscent of Spark's RDDs. Finally, Section 3.3 shows an implementation of k-means clustering which demonstrates computational patterns supported by the MBrace framework (Dzik et al., 2013), a programming system closely related to the function passing model.

\subsection{Higher-Order Operations}

join. Suppose we are given two silos with the following types:

val silo1: SiloRef[List[A]]

val silo2: SiloRef[List[B]] 
as well as two hash functions computing hashes (of type $\mathrm{K}$ ) for elements of type A and type $B$, respectively:

val hashA: $A \Rightarrow K=\ldots$

val hashB: $B \Rightarrow K=\ldots$

The goal is to compute the hash-join of silo1 and silo2 using a higher-order operation hashJoin:

$\operatorname{def}$ hashJoin[A, B, K](s1: SiloRef[List[A]], s2: SiloRef[List[B]], $f: A \Rightarrow K, g: B \Rightarrow K$ )

: SiloRef $[\operatorname{List}[(K,(A, B))]]=$ ???

To implement hashJoin in terms of silos, the types of the two silos first have to be made equal, through initial apply invocations:

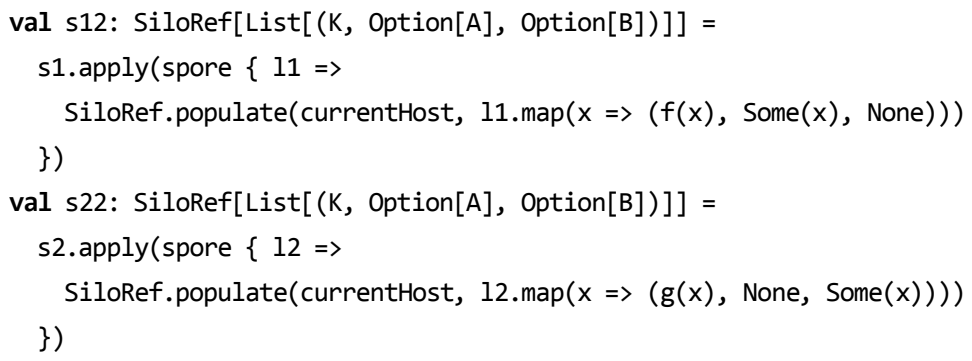

Then, we can use apply to create a new silo which contains the elements of both silo s12 and silo s22: ${ }^{9}$

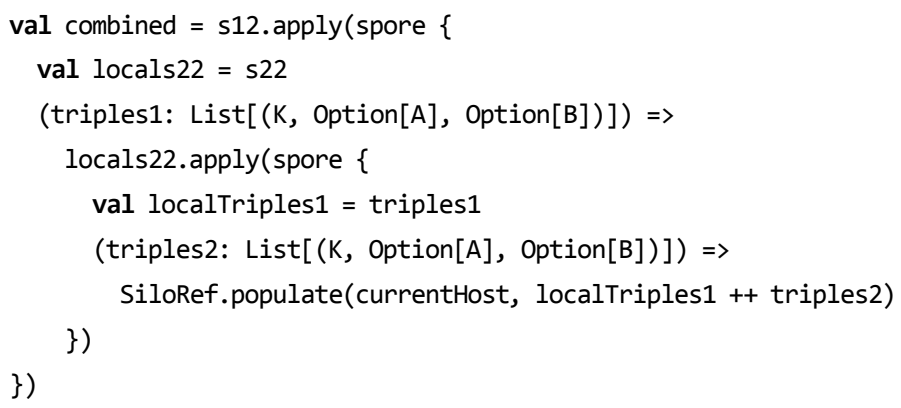

The combined silo contains triples of type (K, Option [A], Option[B]). Using an additional apply, the collection can be sorted by key, and adjacent triples be combined, yielding a SiloRef $[\operatorname{List}[(K,(A, B))]]$ as required.

Partitioning and groupByKey. A groupByKey operation on a group of silos containing collections needs to create multiple result silos, on each host, with ranges of keys supposed to be shipped to destination hosts. These destination hosts are determined using a partitioning function. Our goal, concretely:

val groupedSilos $=$ groupByKey (silos)

9 The expression localTriples1 ++ triples2 denotes the concatenation of lists localTriples1 and triples2. 
Furthermore, we assume that silos.size $=N$ where $N$ is the number of hosts, with hosts $h_{1}, h_{2}$, etc. We assume each silo contains an unordered collection of key-value pairs (a multi-map). Then, groupBykey can be implemented as follows:

- Each host $h_{i}$ applies a partitioning function (example: hash(key) mod $\mathrm{N}$ ) to the keyvalue pairs in its silo, yielding $N$ (local) silos.

- Using apply, each pair of silos containing keys of the same range can be combined and materialized on the destination host.

\subsection{Distributed Collections}

To show that the function passing model is able to serve as a substrate upon which to build different sorts of data-centric distributed frameworks, we have implemented a miniaturized example system that is inspired by Spark's Resilient Distributed Dataset (RDD).

RDDs provide an API for executing data-parallel operations on distributed data. Our simplified RDD implementation provides a collections abstraction distributed using a group of silos. We have implemented some of the operations of Spark's RDD, such as map, reduce, groupBy, and join, in terms of the primitives of the function passing model.

Virtually all methods on RDDs are implemented using the apply method of SiloRef. RDD methods like flatMap or filter that do not require communication across silos are implemented using simple spores which call the corresponding methods of the underlying Scala collections; each spore directly creates its result silo using the populate primitive. (Several examples shown earlier make use of this pattern.) In contrast, methods combining multiple silos, such as join, require nested invocations of the apply method, similar to the example shown in Figure 4.

Below, we show a simple example using our RDD abstraction. The example processes two documents, content and lorem, which are represented as RDDs containing lists of strings:

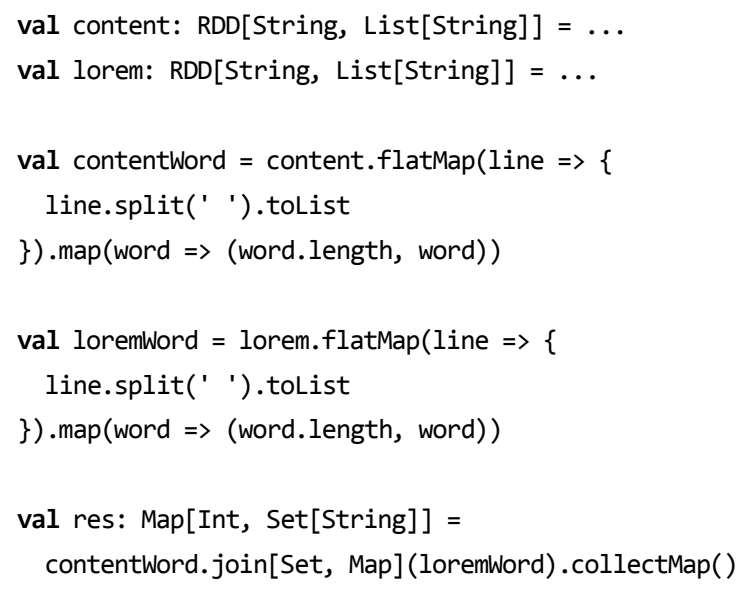

In this example, the closure passed to RDD's flatMap method invoked on contentword and loremword splits each line into a list of words, and flattens everything as a single list. Each word is then mapped to a tuple containing its length and the word. Finally, we do an inner join, which in turn associates each length to the set of words of the same length, 
removing duplicate words in the process. Finally, we collect the final result in a Map using the collectmap method on RDDs. Several other more detailed example programs using RDDs on top of the function passing model are available online. ${ }^{10}$

\subsection{K-Means Clustering}

In order to illustrate how the function passing model supports computation patterns provided by the closely related MBrace (Dzik et al., 2013) framework, we ported an example implementation of k-means clustering. ${ }^{11}$ Below we show an excerpt of the implementation. (See Appendix A.2 for a diagram illustrating various Scala features used in the listing.) Our implementation of distributed k-means clustering using the function passing model is an almost identical port of the version using MBrace written in $\mathrm{F} \sharp$. K-means is an algorithm to categorize data points across $k$ different clusters. It starts with the centroids of the $k$ clusters.

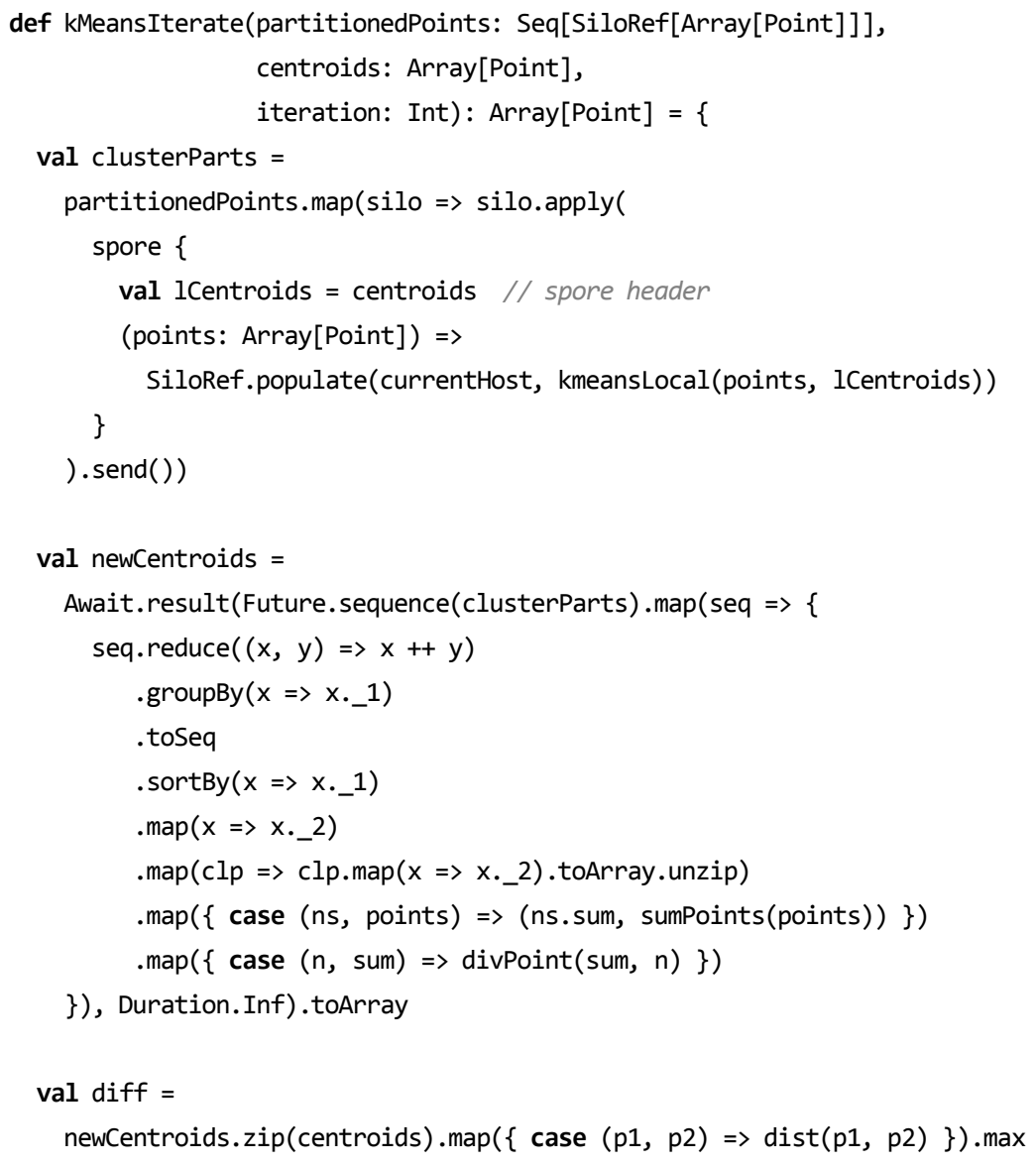

10 See the Git repository at https://github.com/heathermiller/f-p/, branch jfp.

11 See the MBrace website, http://mbrace.io/starterkit/HandsonTutorial.FSharp/examples/ 200-kmeans-clustering-example.html. 


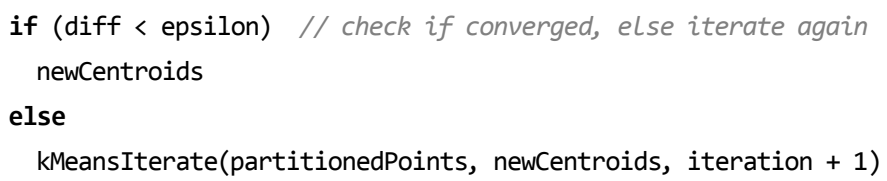

The algorithm proceeds in two steps. It first assigns data points to the closest cluster. Then it assigns to each cluster a new centroid by computing the mean of all points assigned to the cluster. ${ }^{12}$ It stops when the centroids stop changing; if this convergence condition has not been met, the algorithm is called recursively with the updated set of centroids. In the distributed version of k-means clustering, we start with a master node that partitions the points into silos. In each iteration, apply is called on the SiloRef which results in a spore function being applied to the data within the corresponding silo. The spore captures the centroids of the current iteration and uses them to compute the new cluster for its local set of points (using the kmeansLocal function). The results are then sent back to the master node to compute the new centroids, and to verify the algorithm's convergence condition.

\section{Formalization}

We formalize our programming model in the context of a typed lambda calculus. Figure 6 shows the abstract syntax of our core language. Besides standard terms, the language includes terms related to (a) spores, (b) silos, and (c) futures. The spore term creates a new spore. It contains a list of variable definitions, the spore header, and a closure which may only refer to its parameter and variables in the spore header. The populate term initializes a new silo on a given host with a given value. The apply term creates a lineage of silo transformations represented as a silo reference (see below). The send term forces the materialization of the argument silo. The send expression returns a future which is asynchronously completed with the silo's value. The await term waits for the completion of its argument future and returns the future's value. Decentralized identifiers $l$ are used to refer to futures and to identify silos via their lineages (each element in a lineage carries a decentralized identifier).

Values in our language are as expected: besides abstractions and integer literals they include spore values, decentralized identifiers, and silo references. Decentralized identifiers and silo references are not part of the "surface syntax" of our language; they are only introduced by evaluation (see Section 4.1). Silo reference values have the form $\operatorname{Ref}(l, h)$ where $l$ is a lineage and $h$ is a host. Lineages are values of a simple datatype with constructors Mat and Applied. The constructors include all information required for materializing a silo with the result of applying the described transformations. We defer a detailed explanation of the transformations described by a lineage to the following Section 4.1.

In addition to standard function types and Int and Unit types, the language has types for spores, futures, silo references, and hosts (see Figure 7). A spore type $T \Rightarrow T^{\prime}\{$ type $\mathscr{C}=$ $\bar{T}$ \} includes the types $\bar{T}$ of the variables declared in the header of the spore.

12 Simply for the sake of illustration we assume no node failures in this computation. 


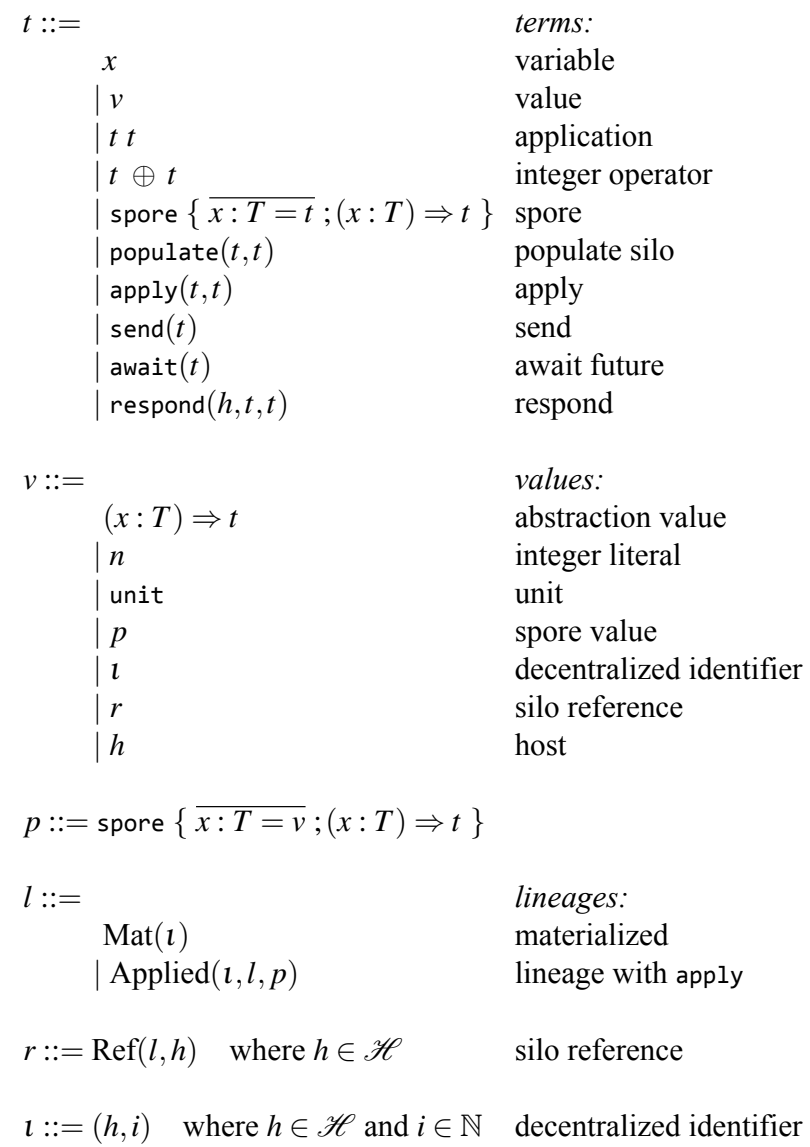

Fig. 6. Abstract syntax of core language. Integer operators are represented using $\oplus \in\{+,-, *, /\}$.

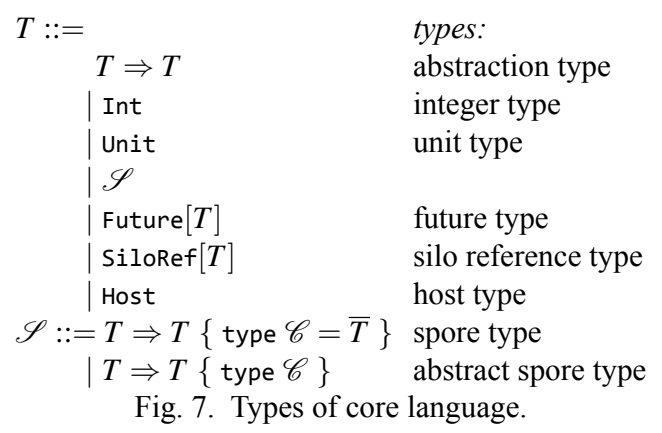

\subsection{Operational Semantics}

In the following we present a small-step operational semantics of the introduced core language. The semantics is clearly stratified into a local (sequential) layer and a distributed (concurrent) layer. Importantly, this means our programming model can benefit from existing reasoning techniques for sequential programs. Program transformations that are correct for sequential programs are also correct for distributed programs. Our programming model shares this property with some existing approaches (Peyton Jones et al., 1996). 


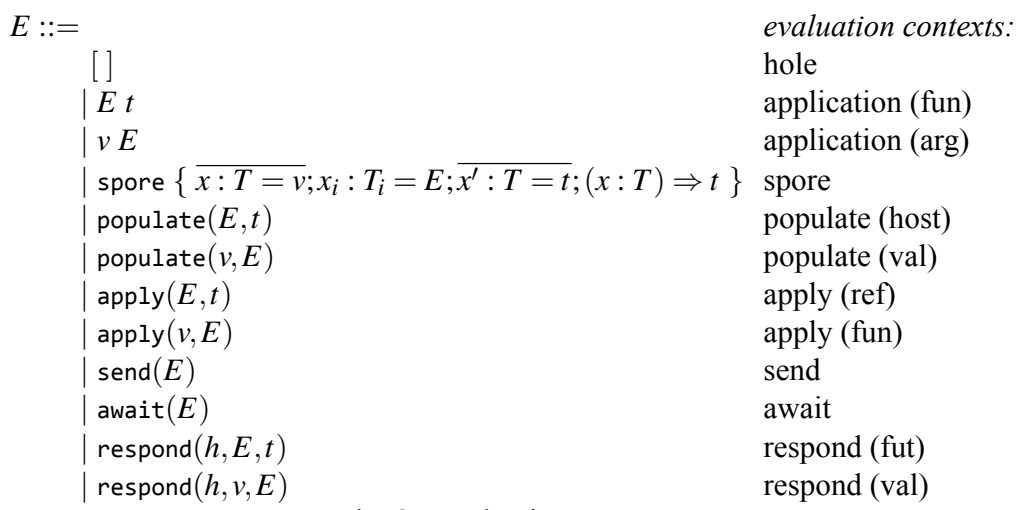

Fig. 8. Evaluation contexts.

$$
\begin{aligned}
& \text { R-INTOP }
\end{aligned}
$$

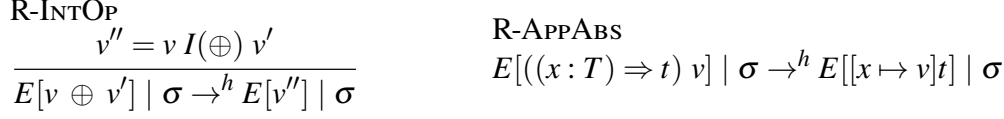

$$
\begin{aligned}
& \text { R-APPSPORE } \\
& E[(\text { spore }\{\overline{x: T=v} ;(x: T) \Rightarrow t\}) v]\left|\sigma \rightarrow^{h} E[\overline{[x \mapsto v]}[x \mapsto v] t]\right| \sigma \\
& \frac{\sigma(l)=v}{E[\operatorname{await}(l)]\left|\sigma \rightarrow^{h} E[v]\right| \sigma} \\
& \text { R-APPLY } \\
& \frac{r=\operatorname{Ref}\left(l, h^{\prime}\right) \quad l^{\prime}=\operatorname{Applied}((h, i), l, p) \quad i \text { fresh }}{E[\operatorname{apply}(r, p)]\left|\sigma \rightarrow^{h} E\left[\operatorname{Ref}\left(l^{\prime}, h^{\prime}\right)\right]\right| \sigma}
\end{aligned}
$$

Fig. 9. Local reduction.

The semantics is based on two reduction relations for (a) local reduction of terms and (b) distributed reduction of sets of hosts. The reduction relations use the definition of evaluation contexts shown in Figure 8. Evaluation contexts capture the notion of the "next subterm to be evaluated." Following a standard approach (Pierce, 2002), we write $E[t]$ for the term obtained by replacing the hole in evaluation context $E$ with term $t$.

Figure 9 shows the rules for local reduction. The local reduction relation has the form $E[t]\left|\sigma \rightarrow^{h} E\left[t^{\prime}\right]\right| \sigma^{\prime}$ with stores $\sigma$ and $\sigma^{\prime}$. Stores are required for the dynamic allocation of silos. A store $\sigma$ is a partial function mapping decentralized identifiers $t$ to values $v$. The annotation with host $h$ is used for creating decentralized identifiers $l=(h, i)$ for lineages. Rule R-INTOP reduces integer operator applications; function $I$ interprets the operator symbol $\oplus$, mapping it to an actual operation on integer values. (The definition of $I$ is trivial and standard, and thus omitted.) Rule R-AppABs is completely standard. Analogous to rule RAppAbs, rule R-APPSPORE describes the application of a spore value to an argument value. Rule R-AwaIt reduces await $(l)$ to $v$ if future $l$ is already completed with $v$ in store $\sigma$.

Rule R-AppLy creates a lineage using the constructor Applied. The new lineage has a fresh identifier $(h, i)$ which uniquely identifies the corresponding (logical) silo. The spore value $p$ is stored in the new lineage; this enables a materialization of the silo identified by $(h, i)$ using parent lineage $l$ and spore $p$.

Distributed reduction. The distributed reduction rules use helper functions host, id, and parent, which are defined as follows: 


$$
m::=\begin{array}{ll}
\operatorname{Req}(h, r, \imath) & \text { messages: } \\
\mid \operatorname{Res}(\boldsymbol{l}, v) & \text { response }
\end{array}
$$

Fig. 10. Messages.

Definition 4.1 (Host identifier) The host identifier of a silo reference.

$$
h o s t(\operatorname{Ref}(l, h)):=h
$$

Definition 4.2 (Lineage identifier) The identifier of a lineage.

$$
i d(l):= \begin{cases}\imath & \text { if } l=\operatorname{Mat}(\imath) \\ \imath & \text { if } l=\operatorname{Applied}\left(\boldsymbol{\imath},{ }_{-},{ }_{-}\right)\end{cases}
$$

Definition 4.3 (Lineage parent) The parent of a lineage.

$$
\operatorname{parent}(l):= \begin{cases}l^{\prime} & \text { if } l=\operatorname{Applied}\left({ }_{-}, l^{\prime},{ }_{-}\right)\end{cases}
$$

The distributed reduction relation has the form $H\left|M \rightarrow H^{\prime}\right| M^{\prime}$ where $H, H^{\prime}$ are sets of hosts and $M, M^{\prime}$ are multisets of messages. A host is a machine that executes a computation, and that can store silos in its local memory. In our formal model a host is represented as a pair $(t, \sigma)^{h}$ consisting of a term $t$ which is the executed computation, and a partial function $\sigma$ which is the local store. Note that each host has a unique host identifier; for instance, the host identifier of host $(t, \sigma)^{h}$ is $h$. When it is clear from the context we use the terms "host" and "host identifier" interchangeably. The multisets $M, M^{\prime}$ model message sends that are "in flight;" a message send $h \leftarrow m$ expresses the sending of message $m$ to host $h$. If $h \leftarrow m \in M$ then message $m$ has not yet been delivered to $h$ (the message is still in transit).

As shown in Figure 10 there are two kinds of messages. A message of the form $\operatorname{Req}(h, r, \imath)$ requests the value of silo $r$ to be sent to host $h$ for materialization of identifier $l$. A message of the form $\operatorname{Res}(l, v)$ represents the corresponding response, containing the identifier $t$ to be materialized using value $v$.

Figure 11 shows the distributed reduction rules.

Rule R-Local reduces a host $h$ chosen non-deterministically from the set of hosts (the rule "schedules" host $h$ for execution). Term $t$ of host $h$ is reduced according to the local reduction rules. Thus, no communication is taking place; this means that the multiset of in-flight messages $M$ remains unchanged.

Rule R-SEND reduces a term send $(r)$ on host $h$. The reduction initiates the materialization of silo $r$ by sending a message $m=\operatorname{Req}(h, r, i d(l))$ to the host of $r, h^{\prime}$. Thus, the message send $h^{\prime} \leftarrow m$ is added to the resulting multiset of in-flight messages $M^{\prime}$. The send term itself is reduced to the identifier of $r$ 's lineage, $i d(l)$.

Rule R-Populate asynchronously populates a new silo on host $h^{\prime}$ with value $v$ by sending message $\operatorname{Res}(l, v)$ to $h^{\prime}$. Note that the fresh decentralized identifier $l=(h, i)$ is already created on host $h$, which does not require any communication. The new lineage $l$ is materialized under identifier $l$, represented using the lineage value $\operatorname{Mat}(l)$.

Rule R-RESPOND reduces a term respond $\left(h^{\prime}, l, v\right)$ by sending a response message $\operatorname{Res}(l, v)$ to $h^{\prime}$.

Rule R-Process models the processing of a message $m$ taken from the multiset $M$ of in-flight messages. Note that this rule is only enabled if $h$ is suspended, waiting for a silo $\imath$ to be materialized in local store $\sigma$. A host is suspended if its current term has the form $E[$ await $(l)]$ and $\imath \notin \operatorname{dom}(\sigma)$ (there is no mapping for $\imath$ in store $\sigma$ ). The premise $\imath \notin \operatorname{dom}(\sigma)$ 


$$
\begin{aligned}
& \text { R-LocaL } \\
& \left\{(t, \sigma)^{h}\right\} \cup H\left|M \rightarrow^{h} t^{\prime}\right| \sigma^{\prime} \\
& \left.\left(^{\prime}, \sigma^{\prime}\right)^{h}\right\} \cup H \mid M
\end{aligned}
$$

R-SEND

$$
\frac{r=\operatorname{Ref}\left(l, h^{\prime}\right) \quad m=\operatorname{Req}(h, r, i d(l)) \quad M^{\prime}=M \uplus\left\{h^{\prime} \leftarrow m\right\}}{\left\{(E[\operatorname{send}(r)], \sigma)^{h}\right\} \cup H\left|M \rightarrow\left\{(E[i d(l)], \sigma)^{h}\right\} \cup H\right| M^{\prime}}
$$

R-Populate
$\frac{l=(h, i) \quad i \text { fresh } \quad l=\operatorname{Mat}(l) \quad M^{\prime}=M \uplus\left\{h^{\prime} \leftarrow \operatorname{Res}(l, v)\right\}}{\left\{\left(E\left[\text { populate }\left(h^{\prime}, v\right)\right], \sigma\right)^{h}\right\} \cup H\left|M \rightarrow\left\{\left(E\left[\operatorname{Ref}\left(l, h^{\prime}\right)\right], \sigma\right)^{h}\right\} \cup H\right| M^{\prime}}$

R-RESPOND

$$
\frac{M^{\prime}=M \uplus\left\{h^{\prime} \leftarrow \operatorname{Res}(l, v)\right\}}{\left\{\left(E\left[\text { respond }\left(h^{\prime}, l, v\right)\right], \sigma\right)^{h}\right\} \cup H \mid M \rightarrow\left\{(E[\text { unit }], \sigma)^{h}\right\} \cup H \mid M^{\prime}}
$$

R-Process

$$
\begin{gathered}
\frac{l \notin \operatorname{dom}(\sigma) \quad M=M^{\prime} \uplus\{h \leftarrow m\} \quad \operatorname{process}(h, m, \sigma)=\left(t, M^{\prime \prime}, \sigma^{\prime}\right)}{\left\{(E[\operatorname{await}(\imath)], \sigma)^{h}\right\} \cup H\left|M \rightarrow\left\{\left(E[t ; \operatorname{await}(\imath)], \sigma^{\prime}\right)^{h}\right\} \cup H\right| M^{\prime} \uplus M^{\prime \prime}} \\
\text { R-Process-VAL } \\
\frac{M=M^{\prime} \uplus\{h \leftarrow m\} \quad \operatorname{process}(h, m, \sigma)=\left(t, M^{\prime \prime}, \sigma^{\prime}\right)}{\left\{(v, \sigma)^{h}\right\} \cup H\left|M \rightarrow\left\{\left(t, \sigma^{\prime}\right)^{h}\right\} \cup H\right| M^{\prime} \uplus M^{\prime \prime}}
\end{gathered}
$$

Fig. 11. Distributed reduction.

is important, since otherwise rule R-Local would also be enabled. Rule R-Process-VAL is analogous to rule R-Process for the case where a host $h$ is unable to reduce its term further.

The actual message processing logic is factored out into function process which we discuss in the following. In general, processing a message $m$ on host $h$ in store $\sigma$ results in (a) a term $t$ to be evaluated by $h$, (b) a set of new in-flight messages $M^{\prime}$, and (c) an updated store $\sigma^{\prime}$.

Definition 4.4 (Message processing) The function process $\in \mathscr{H} \times m \times S \rightarrow t \times \mathscr{P}(\mathscr{H} \times$ $m) \times S$ handles the processing of a message resulting in a term to be evaluated, a set of messages, and an updated store; here, $S=\mathscr{H} \times \mathbb{N} \rightarrow v$ is the type of a store. process is defined in Fig. 12.

The process function must handle the two kinds of messages, $\operatorname{Req}\left(h^{\prime}, r, \imath\right)$ and $\operatorname{Res}(l, v)$. Processing a message of the form $\operatorname{Res}(l, v)$ means that a host is responding with the value $v$ of a silo whose materialization has been requested by the current host $h$ to complete its future $l$. Thus, it suffices to create an updated store $\sigma^{\prime}$ which maps $l$ to $v$ (rule Proc-REs).

Processing a message of the form $\operatorname{Req}\left(h^{\prime}, r, l\right)$ means that host $h^{\prime}$ requests the value of silo $r$ in order to complete a future with identifier 2 . In the absence of caching/persisting, this requires the receiver of the request to materialize the silo whose value is requested. Section 4.4 introduces a refinement of these rules to enable caching, thereby avoiding repeated materializations of the same silo.

The requested silo $r=\operatorname{Ref}(l, h)$ is materialized using its lineage $l$. Rule Proc-ReQParent handles the case where $r$ 's parent $i d\left(l^{\prime}\right)$ is not yet materialized $\left(l^{\prime}\right.$ is the lineage of $r$ 's parent). In this case, process returns the term send $\left(\operatorname{Ref}\left(l^{\prime}, h\right)\right)$; this causes 
Proc-Res
\[ \sigma^{\prime}=[l \mapsto v] \sigma \]
$\operatorname{process}(h, \operatorname{Res}(l, v), \sigma)=\left(\right.$ unit $\left., \emptyset, \sigma^{\prime}\right)$

Proc-ReqParent

$\frac{r=\operatorname{Ref}(l, h) \quad l^{\prime}=\operatorname{parent}(l) \quad i d\left(l^{\prime}\right) \notin \operatorname{dom}(\sigma) \quad M=\left\{h \leftarrow \operatorname{Req}\left(h^{\prime}, r, l\right)\right\}}{\operatorname{process}\left(h, \operatorname{Req}\left(h^{\prime}, r, l\right), \sigma\right)=\left(\operatorname{send}\left(\operatorname{Ref}\left(l^{\prime}, h\right)\right), M, \sigma\right)}$

Proc-ReqMat1

$\frac{r=\operatorname{Ref}(l, h) \quad l=\operatorname{Mat}\left(\iota^{\prime}\right) \quad \sigma\left(\iota^{\prime}\right)=v \quad M=\left\{h^{\prime} \leftarrow \operatorname{Res}(\boldsymbol{l}, v)\right\}}{\operatorname{process}\left(h, \operatorname{Req}\left(h^{\prime}, r, \imath\right), \sigma\right)=(\text { unit }, M, \sigma)}$

Proc-ReqMat2

$\frac{r=\operatorname{Ref}(l, h) \quad l=\operatorname{Mat}\left(\imath^{\prime}\right) \quad \iota^{\prime} \notin \operatorname{dom}(\sigma) \quad M=\left\{h \leftarrow \operatorname{Req}\left(h^{\prime}, r, \imath\right)\right\}}{\operatorname{process}\left(h, \operatorname{Req}\left(h^{\prime}, r, \imath\right), \sigma\right)=(\text { unit }, M, \sigma)}$

Proc-ReqApply

$\frac{r=\operatorname{Ref}(l, h) \quad l=\operatorname{Applied}\left(l^{\prime}, l^{\prime}, p\right) \quad \sigma\left(i d\left(l^{\prime}\right)\right)=v \quad t=\operatorname{respond}\left(h^{\prime}, l, \text { await }(\operatorname{send}(p v))\right)}{\operatorname{process}\left(h, \operatorname{Req}\left(h^{\prime}, r, l\right), \sigma\right)=(t, \emptyset, \sigma)}$

Fig. 12. Message processing.

host $h$ to request the materialization of the parent silo $\operatorname{Ref}\left(l^{\prime}, h\right)$ before any other message is processed. Note that a silo is always materialized on the same host as its parent silo; therefore, the hosts of $r$ and its parent are guaranteed to be the same. The original request $\operatorname{Req}\left(h^{\prime}, r, \imath\right)$ is included in the new in-flight messages $M$, so that it is eventually handled, namely when $r$ 's parent is materialized.

Rules Proc-ReqMat1 and Proc-ReqMat2 handle the case where $r$ does not have a parent. In the case of Proc-ReQMAT1, the materialization of $r$ is just the value $v$ of its materialized lineage $\operatorname{Mat}\left(\iota^{\prime}\right)$; thus, a response $\operatorname{Res}(l, v)$ is sent to host $h^{\prime}$. In the case of Proc-ReqMat2, lineage Mat $\left(l^{\prime}\right)$ is not yet materialized (i.e., host $h$ is still waiting for a message $\left.\operatorname{Res}\left(\iota^{\prime}, v^{\prime}\right)\right)$; thus, the original request $\operatorname{Req}\left(h^{\prime}, r, \imath\right)$ sent to $h$ is re-sent, to be processed again later.

Rule PROC-REQAPPLY is only enabled when the requested silo $r$ can be materialized using its lineage $l$; in particular, $r$ 's parent must be materialized. In this case the lineage of $r$ begins with an Applied constructor. The materialization of $r$ consists of the result of applying the spore $p$ provided by the lineage to the value $v$ of the parent silo. Evaluating the application $p v$ requires multiple reduction steps in general. Therefore, rule Proc-REQApPLY returns a term containing the application $p v$ for evaluation by host $h$. However, note that $p v$ reduces to a silo reference. Therefore, the value of silo $p v$ is obtained by requesting its materialization (using send $(p v)$ ) and waiting for the completion of the returned future (using await). Finally, when the future is resolved, a response $\operatorname{Res}\left(\imath, v^{\prime}\right)$ (for some value $v^{\prime}$ ) is sent to host $h^{\prime}$ by evaluating the respond term.

\subsection{Type Assignment}

Type assignment is based on a judgment of the form $\Gamma ; \Sigma \vdash t: T$ which assigns term $t$ type $T$. $\Gamma$ is a standard type environment which maps variables $x$ to types $T$; $\Sigma$ is a store typing which 


$$
\begin{aligned}
& \text { T-VAR T-InT } 2 \text { T-InTOP } \\
& \frac{x: T \in \Gamma}{\Gamma ; \Sigma \vdash x: T} \quad \frac{n \text { integer literal }}{\Gamma ; \Sigma \vdash n: \text { Int }} \quad \frac{\Gamma ; \Sigma \vdash t: \text { Int } \quad \Gamma ; \Sigma \vdash t^{\prime}: \text { Int }}{\Gamma ; \Sigma \vdash t \oplus t^{\prime}: \text { Int }}
\end{aligned}
$$

T-AppSPORE

$$
\frac{\Gamma ; \Sigma \vdash t: T \Rightarrow T^{\prime}\{\text { type } \mathscr{C}=\bar{T}\} \quad \Gamma ; \Sigma \vdash t^{\prime}: T}{\Gamma ; \Sigma \vdash\left(t t^{\prime}\right): T^{\prime}}
$$

T-APPLY

T-SILOREF$$
\frac{\Sigma(i d(l))=T \quad \Sigma \vdash \operatorname{Ref}(l, h)}{\Gamma ; \Sigma \vdash \operatorname{Ref}(l, h): \operatorname{SiloRef}[T]}
$$

$$
\frac{\Gamma ; \Sigma \vdash t: \text { SiloRef }[T] \quad \Gamma ; \Sigma \vdash t^{\prime}:\left(T \Rightarrow \operatorname{SiloRef}\left[T^{\prime}\right]\{\text { type } \mathscr{C}=\bar{T}\}\right)}{\Gamma ; \Sigma \vdash \operatorname{apply}\left(t, t^{\prime}\right): \operatorname{SiloRef}\left[T^{\prime}\right]} \quad \frac{\Gamma ; \Sigma \vdash t: \operatorname{SiloRef}[T]}{\Gamma ; \Sigma \vdash \operatorname{send}(t): \text { Future }[T]}
$$

\begin{tabular}{|c|c|c|c|}
\hline $\begin{array}{l}\text { S-INT } \\
\text { serializable(Int) }\end{array}$ & $\begin{array}{l}\text { S-Unit } \\
\text { serializable(Unit) }\end{array}$ & $\begin{array}{l}\text { S-Host } \\
\text { serializable (Host) }\end{array}$ & $\begin{array}{l}\text { S-SiLoReF } \\
\text { serializable }(\text { SiloRef }[T])\end{array}$ \\
\hline \multicolumn{4}{|c|}{ S-SPORE } \\
\hline & serializable( & $T^{\prime}\{$ type $\mathscr{C}=\bar{T}\}$ & \\
\hline
\end{tabular}

T-AwAIT $\frac{\Gamma ; \Sigma \vdash t: \text { Future }[T]}{\Gamma ; \Sigma \vdash \text { await }(t): T}$
T-IDENT

T-RESPOND

$\frac{\Sigma(\imath)=T}{\Gamma ; \Sigma \vdash \imath: \text { Future }[T]}$
T-SEND

T-Populate $\frac{\Gamma ; \Sigma \vdash t: \text { Host } \quad \Gamma ; \Sigma \vdash t^{\prime}: T \quad \text { serializable }(T)}{\Gamma ; \Sigma \vdash \text { populate }\left(t, t^{\prime}\right): \operatorname{SiloRef}[T]}$

Fig. 13. Type assignment.

Fig. 14. Serializable types.

maps identifiers $\imath$ to types $T$. Figure 13 shows the rules for type assignment. Rules T-VAR, T-ABs, and T-App are unchanged compared to a standard typed lambda calculus (Pierce, 2002). Rules T-INT and T-INTOP assign types to integer literals and applications of integer operators, respectively.

Rule T-SPORE assigns a type to spore literals. Importantly, the body of the spore's closure, $t$, must be well-typed in a type environment containing only the closure parameter $x$ and the variables $\bar{x}$ in the spore's header, as well as an empty store typing. Furthermore, the types of captured variables as well as the result type $T^{\prime}$ must be serializable. The predicate serializable is defined in Figure 14. These constraints ensure that spore values are always independent of the environment of the creating host. This independence is expressed by the following theorem: 


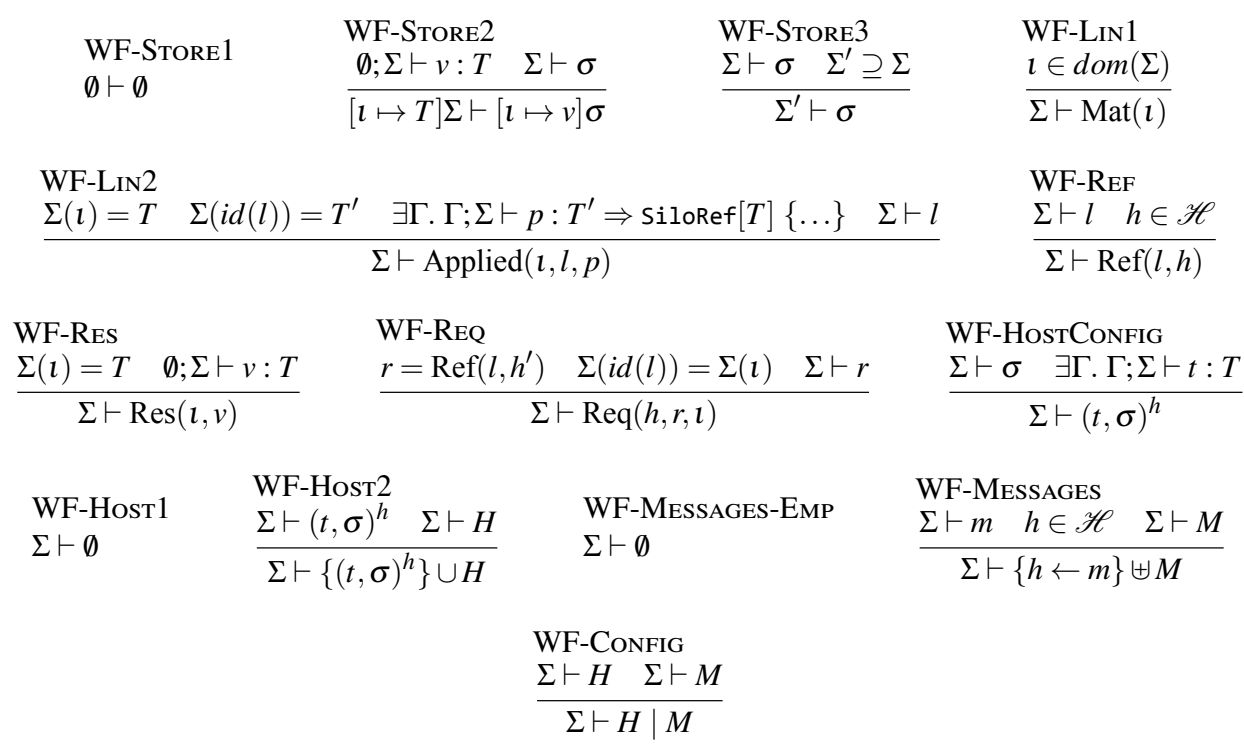

Fig. 15. Well-formedness.

Theorem 4.1 (Serializable Values) If $\Gamma ; \Sigma \vdash v: T$ and serializable( $T$ ) then $\emptyset ; \Sigma \vdash v: T$.

Proof

By induction on the derivation of $\Gamma ; \Sigma \vdash v: T$. See Appendix B.1.

Rule T-APPSPore is analogous to rule T-ApP. Rules T-Populate and T-ApPLY are straightforward; note that apply is polymorphic in the types of the captured variables of its spore argument type. Rules T-SEND and T-AwAIT are entirely unsurprising. Rules T-IDENT and T-SiLoRef are the only rules that use the store typing $\Sigma$. The type of an identifier $\imath$ has the form Future $[T]$ where type $T$ is looked up in the store typing. Rule T-SiLoRef is analogous; additionally, it requires the silo reference $\operatorname{Ref}(l, h)$ to be well-formed in $\Sigma$ (see below).

\subsection{Well-Formed Configurations}

Figure 15 shows the rules for well-formed configurations. These rules are essential for establishing subject reduction (see Section 5.1). Rules WF-STORE1-3 are straightforward. Rule WF-Lin 1 requires $\Sigma$ to be defined for the identifier of a materialized lineage Mat $(l)$. Rule WF-Lin2 requires the types of $\imath$ and $i d(l)$ given by the store typing $\Sigma$ to be consistent with the corresponding type of spore $p$. Parent lineage $l$ must be well-formed in $\Sigma$. Rule WF-REF extends well-formedness of lineages to silo references. Rules WF-REs and WFREQ specify well-formedness of messages in $\Sigma$. The remaining rules lift well-formedness to host configurations (WF-HostConfig), sets of hosts (WF-Host1-2), multisets of messages (WF-Messages-Emp, WF-Messages), and configurations (WF-Config), respectively.

\subsection{Persist and Unpersist}

As explained in Section 2.4, silos may be cached in memory to avoid repeated materialization, which may be expensive for large data sets. The design is inspired by the popular 


$$
\begin{aligned}
& t:: \quad \text { terms: } \\
& \text { | persist }(t) \quad \text { persist } \\
& \text { | unpersist }(t) \text { unpersist } \\
& l::=\begin{array}{ll}
\ldots \\
\mid \text { Persist }(\iota, l, f) & \text { lineages: } \\
& \text { lineage with persist }
\end{array} \\
& E::=\quad \text { evaluation contexts: } \\
& \text {... } \\
& \mid \operatorname{persist}(E) \quad \text { persist } \\
& \text { unpersist }(E) \text { unpersist }
\end{aligned}
$$

Fig. 16. Extensions to syntax, lineages, and evaluation contexts for persist/unpersist. $f$ is a binary operator with $f \in\{\cdot \cup \cdot, \backslash \backslash \cdot\}$.

$$
\begin{aligned}
& \text { R-Persist } \\
& \frac{r=\operatorname{Ref}\left(l, h^{\prime}\right) \quad l^{\prime}=\operatorname{Persist}((h, i), l, \cdot \cup \cdot) \quad i \text { fresh }}{E[\operatorname{persist}(r)]\left|\sigma \rightarrow{ }^{h} E\left[\operatorname{Ref}\left(l^{\prime}, h^{\prime}\right)\right]\right| \sigma} \\
& \text { R-UNPERSIST } \\
& \frac{r=\operatorname{Ref}\left(l, h^{\prime}\right) \quad l^{\prime}=\operatorname{Persist}((h, i), l, \cdot \backslash \cdot) \quad i \text { fresh }}{E[\text { unpersist }(r)]\left|\sigma \rightarrow^{h} E\left[\operatorname{Ref}\left(l^{\prime}, h^{\prime}\right)\right]\right| \sigma} \\
& \frac{\sigma(\imath)=(v, P)}{E[\operatorname{await}(l)]\left|\sigma \rightarrow^{h} E[v]\right| \sigma}
\end{aligned}
$$

Fig. 17. Extension to local reduction.

Spark (Zaharia et al., 2010) data processing system. Essentially, a silo $r$ may be persisted using the persist primitive: the call persist $(r)$ immediately returns a new silo reference $r^{\prime}$ whose value, when materialized, is equal to the value of $r$. The lineage of $r^{\prime}$ is equal to that of $r$ except for one additional element: if $l$ is the lineage of silo $r$, then the lineage of $r^{\prime}$ is of the form $l^{\prime}=\operatorname{Persist}(l, l, f)$. As for the Applied constructor $l$ is the identifier of lineage $l^{\prime}$. (As before, $l=(h, i)$ implies that host $h$ has called persist.) The last argument is a binary operator $f \in\{\cdot \cup \cdot, \cdot \backslash \cdot\}$ which toggles between the behavior of persist and unpersist. If $f=\cdot \cup \cdot$ then the lineage Persist $(l, l, f)$ implements the behavior of persist; otherwise, the behavior of unpersist. The extensions to syntax, lineages, and evaluation contexts are summarized in Figure 16.

\subsubsection{Operational semantics}

In order to distinguish between silos that have been persisted and those that have not, we extend stores $\sigma$ to map identifiers $l$ not just to their associated value, but also to a so-called "persist set" $P$; if $\sigma(\imath)=(v, P)$ then $P$ contains all hosts that have persisted silo $\imath$. Stores have thus the following extended type:

Definition 4.5 (Store) $\sigma \in \mathscr{H} \times \mathbb{N} \rightarrow v \times \mathscr{P}(\mathscr{H})$.

As a next step we extend the local reduction relation, as shown in Figure 17. First, we introduce the reduction rules R-PERSIST and R-UNPERSIST to enable the creation of Persist lineages. Second, we adjust reduction rule R-AwaIt to use the extended store. 


$$
\begin{aligned}
& \text { Proc-Res } \\
& \sigma^{\prime}=[\imath \mapsto(v, \emptyset)] \sigma \\
& \overline{\operatorname{process}(h, \operatorname{Res}(l, v), \sigma)=\left(\text { unit }, \emptyset, \sigma^{\prime}\right)}
\end{aligned}
$$

Fig. 18. Extensions to message processing.

The rules for message processing are impacted most. Intuitively, the reason is that the fact whether a silo is (re-)materialized is determined in the context of message processing. Figure 18 shows new or extended rules for message processing. Rule Proc-Res is simply adjusted to the new store definition. Rule Proc-ReqPersist is new; the rule enables processing requests $\operatorname{Req}\left(h^{\prime}, r, l\right)$ in cases where $r$ has a Persist lineage and $r$ 's parent is materialized (otherwise, the above Proc-ReQParent rule would be enabled). Importantly, a silo with lineage Persist $\left(l^{\prime}, l^{\prime}, \star\right)$ has the same value as the parent silo which has identifier $i d\left(l^{\prime}\right)$. Therefore, rule Proc-ReqPersist updates the store to map the identifier of the persisted silo, $l^{\prime}$, to the value of $i d\left(l^{\prime}\right)$ in $\sigma$. In addition, a persist set $P^{\prime}$ is computed based on (1) the persist set $P$ of the parent silo, (2) the host $h^{\prime \prime}$ that created the Persist lineage, and (3) the operator $\star$ provided in the Persist lineage. The latter may either be set union or set difference; accordingly, the persisting host $h^{\prime \prime}$ is either added or removed from the persist set $P$ of the parent of the silo to-be-persisted. In case $h^{\prime \prime}$ is added to the persist set (because host $h^{\prime \prime}$ called persist rather than unpersist), it means that the persist set $P^{\prime}$ is non-empty. As a result, the mapping for $\iota^{\prime}$ remains resident in the store, avoiding re-materialization, as the following rules show.

Rule Proc-Req is also new. The rule is enabled when the requested silo $r$ has already been materialized, i.e., $\sigma(i d(l))=(v, P)$. In this case, a response $\operatorname{Res}(l, v)$ can directly be sent back to the requesting host $h^{\prime}$. Importantly, the rule also checks whether the silo has been persisted, by examining its persist set $P$ in store $\sigma$. Using the consume function, the mapping for identifier id $(l)$ is removed from the updated store $\sigma^{\prime}$ if the persist set $P$ is empty. An empty persist set indicates that the corresponding silo has not been persisted. Concretely, the consume function is defined as follows:

Definition 4.6 (Consume silo) Consume silo referenced by 1 with persist set $P$ in store $\sigma$ consume $(\imath, P, \sigma):= \begin{cases}\sigma-\imath & \text { if } P=\emptyset \\ \sigma & \text { otherwise }\end{cases}$ 


$$
\begin{aligned}
& \text { T-Persist } \\
& \frac{\Gamma ; \Sigma \vdash t: \operatorname{SiloRef}[T]}{\Sigma \vdash \operatorname{persist}(t): \operatorname{SiloRef}[T]} \\
& \text { WF-Store2 } \\
& \frac{\emptyset ; \Sigma \vdash v: T \quad \Sigma \vdash \sigma}{[\imath \mapsto T] \Sigma \vdash[\imath \mapsto(v, P)] \sigma}
\end{aligned}
$$

$$
\begin{aligned}
& \text { T-UnPERsist } \\
& \frac{\Gamma ; \Sigma \vdash t: \operatorname{SiloRef}[T]}{\Gamma ; \Sigma \vdash \text { unpersist }(t): \operatorname{SiloRef}[T]}
\end{aligned}
$$$$
\text { WF-Lin3 }
$$$$
\frac{\Sigma(l)=\Sigma(i d(l)) \quad \Sigma \vdash l}{\Sigma \vdash \operatorname{Persist}(l, l, \star)}
$$

Fig. 19. Extension to type assignment and well-formedness.

Rule Proc-ReqApply is extended to consume the parent silo in case it has not been persisted.

Figure 19 shows the required extensions to type assignment and well-formedness. The type rules T-Persist and T-UnPERsist are straightforward. Rule WF-Store2 is simply adjusted to the extended store definition. Rule WF-Lin3 defines well-formedness for Persist lineages: the store typings for $l$ and $i d(l)$ must be equal and parent lineage $l$ must be wellformed.

\section{Correctness Properties}

\subsection{Subject Reduction}

This section establishes a subject reduction theorem for the presented core language. The complete proof is provided in the appendix; here, we restrict ourselves to summarizing the main results.

Lemma 5.1 (Substitution) If $\Gamma, x: T^{\prime} ; \Sigma \vdash t: T$ and $\Gamma ; \Sigma \vdash v: T^{\prime}$ then $\Gamma ; \Sigma \vdash[x \mapsto v] t: T$.

Proof

By induction on the derivation of $\Gamma, x: T^{\prime} ; \Sigma \vdash t: T$.

Theorem 5.2 (Subject Reduction)

1. If $\Gamma ; \Sigma \vdash t: T$, $\Sigma \vdash \sigma$, and $t\left|\sigma \rightarrow^{h} t^{\prime}\right| \sigma^{\prime}$ then $\Gamma ; \Sigma^{\prime} \vdash t^{\prime}: T$ and $\Sigma^{\prime} \vdash \sigma^{\prime}$ for some $\Sigma^{\prime} \supseteq \Sigma$.

2. If $\Sigma \vdash H \mid M$ and $H\left|M \rightarrow H^{\prime}\right| M^{\prime}$ then $\Sigma^{\prime} \vdash H^{\prime} \mid M^{\prime}$ for some $\Sigma^{\prime} \supseteq \Sigma$.

Proof

Part 1: by induction on the derivation of $t\left|\sigma \rightarrow^{h} t^{\prime}\right| \sigma^{\prime}$. Part 2: by induction on the derivation of $H\left|M \rightarrow H^{\prime}\right| M^{\prime}$. See Appendix B.2 for the complete proof.

\subsection{Progress}

This section formulates progress properties. The main Theorem 5.5 states that materialization requests are satisfied after a finite number of reduction steps in so-called "responsive configurations."

In the following we assume a fair scheduling property which ensures that in a wellformed configuration $H \mid M$, each message $h \leftarrow m \in M$ is eventually received by host $h$. Fair scheduling is also assumed in other models of distributed computing like actors (Agha, 1986; Agha et al., 1997). Formally, fair scheduling is defined as follows: 
Definition 5.1 (Fair Scheduling) Let $\Sigma \vdash H \mid M$ and $h \leftarrow m \in M$ where $\Sigma \vdash m$.

Then $H\left|M \rightarrow{ }^{*} H^{\prime}\right| M^{\prime} \rightarrow H^{\prime \prime} \mid M^{\prime \prime}$ after a finite number of reduction steps, and $H^{\prime} \mid M^{\prime} \rightarrow$ $H^{\prime \prime} \mid M^{\prime \prime}$ by rule R-Process or R-Process-VaL such that $M^{\prime}=M_{\text {old }} \uplus\{h \leftarrow m\},(t, \sigma)^{h} \in H^{\prime}$, process $(h, m, \sigma)=\left(t^{\prime}, M_{\text {new }}, \sigma^{\prime}\right)$, and $M^{\prime \prime}=M_{\text {old }} \uplus M_{\text {new }}$.

Although our focus is the establishment of desirable progress properties for distributed reduction, it is necessary to consider the following strong normalization property of singlehost reductions. For this, we consider the reduction relation $\rightsquigarrow$ defined as the subset of the reduction relation $\rightarrow$ excluding reduction rules R-Process and R-Process-VAL.

Lemma 5.3 (Single-Host Strong Normalization) Let $\Sigma \vdash H \mid M$ where $H=\left\{(t, \sigma)^{h}\right\} \cup$ $H^{\prime}$. Then $H\left|M \rightsquigarrow^{*}\left\{\left(t^{\prime}, \sigma^{\prime}\right)^{h}\right\} \cup H^{\prime}\right| M^{\prime}$ after a finite number of reduction steps and either $t^{\prime}$ is a value or $t^{\prime}=E[$ await $(l)]$.

Note that in the above reduction, only a single host $h$ is reduced. Furthermore, the set of in-flight messages may change, e.g., by applying rule R-SEND. A proof of Lemma 5.3 is outside the scope of this paper. However, our core language is, fundamentally, not more expressive than the simply typed lambda calculus, for which strong normalization holds.

As a prerequisite for the establishment of our main progress theorem, we introduce a small amount of bookkeeping into the reduction relations. The aim is to keep track of silo references created during reduction. Importantly, this additional bookkeeping information does not introduce any change in the semantic behavior-the information can be erased without affecting the run-time semantics in any way.

The augmented local reduction relation has the form $E[t]\left|\sigma \rightarrow^{h} E\left[t^{\prime}\right]\right| \sigma^{\prime} \mid R$ where $R$ is either the empty set or a singleton set containing the created silo reference. Rule R-APPLY is the only rule resulting in a non-empty set of created references:

$$
\begin{aligned}
& \text { R-APPLY } \\
& \frac{r=\operatorname{Ref}\left(l, h^{\prime}\right) \quad l^{\prime}=\operatorname{Applied}((h, i), l, p) \quad i \text { fresh } \quad r^{\prime}=\operatorname{Ref}\left(l^{\prime}, h^{\prime}\right)}{E[\operatorname{apply}(r, p)]\left|\sigma \rightarrow^{h} E\left[r^{\prime}\right]\right| \sigma \mid\left\{r^{\prime}\right\}}
\end{aligned}
$$

The augmented distributed reduction relation has the form $H|M| R \rightarrow H^{\prime}\left|M^{\prime}\right| R^{\prime}$ where $R$ is the set of references already existing before performing the reduction step and $R^{\prime}$ is the set of references existing after performing the reduction step. Rules R-LocAL and R-Populate are the only rules resulting in a set of references $R^{\prime}$ where $R^{\prime} \neq R$ is possible:

$$
\begin{aligned}
& \text { R-Local } \\
& \qquad\left\{(t, \sigma)^{h}\right\} \cup H|M| M \rightarrow^{h} t^{\prime}\left|\sigma^{\prime}\right| R^{\prime} \\
& \left\{\left(t^{\prime}, \sigma^{\prime}\right)^{h}\right\} \cup H|M| R \cup R^{\prime}
\end{aligned}
$$

$$
\begin{aligned}
& \begin{array}{l}
\text { R-Populate } \\
l=(h, i) \quad i \text { fresh } \quad l=\operatorname{Mat}(l) \quad M^{\prime}=M \uplus\left\{h^{\prime} \leftarrow \operatorname{Res}(l, v)\right\} \quad r^{\prime}=\operatorname{Ref}\left(l, h^{\prime}\right)
\end{array} \\
& \left\{\left(E\left[\text { populate }\left(h^{\prime}, v\right)\right], \sigma\right)^{h}\right\} \cup H|M| R \rightarrow\left\{\left(E\left[r^{\prime}\right], \sigma\right)^{h}\right\} \cup H\left|M^{\prime}\right| R \cup\left\{r^{\prime}\right\}
\end{aligned}
$$

Finally, the extension of well-formed configurations is straightforward:

$$
\begin{array}{ll}
\text { WF-CONFIG } & \text { WF-REFS } \\
\frac{\Sigma \vdash H \quad \Sigma \vdash M \quad \Sigma \vdash R}{\Sigma \vdash H|M| R} & \frac{\sum \vdash r \quad \Sigma \vdash R}{\Sigma \vdash\{r\} \cup R}
\end{array}
$$


Using the augmented reduction rules we introduce a responsiveness property, responsive configurations. Informally, in a responsive configuration requesting (the materialization of) any previously created silo reference results in a corresponding response after a finite number of reduction steps. The property is defined as follows:

Definition 5.2 (Responsive Configuration) A configuration $\Sigma \vdash H|M| R$ is responsive, written Responsive $(H, M, R)$, iff

$\forall r=\operatorname{Ref}(l, h) \in R .\left(m=\operatorname{Req}\left(h^{\prime}, r, \imath\right) \wedge \Sigma \vdash m\right) \Longrightarrow H|M \uplus\{h \leftarrow m\}| R \rightarrow^{*} H^{\prime} \mid M^{\prime} \uplus$ $\left\{h^{\prime} \leftarrow \operatorname{Res}(l, v)\right\} \mid R^{\prime}$ after a finite number of reduction steps.

The following lemma ensures that the ability to materialize a silo after a finite number of reduction steps is preserved under reduction.

Lemma 5.4 (Responsiveness) Let $\Sigma \vdash H|M| R \cup \hat{R}$ and Responsive $(H, M, \hat{R})$. If $H|M| R \cup \hat{R} \rightarrow H^{\prime}\left|M^{\prime}\right| R^{\prime} \cup \hat{R}$ then Responsive $\left(H^{\prime}, M^{\prime}, \hat{R}\right)$.

Proof Sketch

By induction on the derivation of $H|M| R \cup \hat{R} \rightarrow H^{\prime}\left|M^{\prime}\right| R^{\prime} \cup \hat{R}$ with case analysis of the last applied rule, using Def. 5.1 and Lemma 5.3.

We are now ready to introduce the main progress theorem. Theorem 5.5 states that finite materialization of silos is a universal property of our core language.

Theorem 5.5 (Finite Materialization) Let $\Sigma \vdash H|M| R$ such that Responsive $(H, M, R)$. If $H|M| R \rightarrow H^{\prime}\left|M^{\prime}\right| R^{\prime}$ then Responsive $\left(H^{\prime}, M^{\prime}, R^{\prime}\right)$.

Proof

See Appendix B.3.

\section{Related Work}

Alice ML (Rossberg et al., 2004) is an extension of Standard ML which adds a number of important features for distributed programming such as futures and proxies. The design leading up to the function passing model has incorporated many similar ideas, such as typesafe, generic and platform-independent pickling. In Alice, functions intend to be mobile. Only those functions which capture (either directly or indirectly) local resources remain stationary. In the case of functions that must remain stationary, it is possible to send proxies, mobile wrappers for functions. Sending a proxy will not transfer the wrapped function; instead, when a proxy function is applied, the call is forwarded by the system to the original site as a remote invocation (pickling arguments and result appropriately). In F-P, however, functions are not wrapped in proxies but sent directly. Thus, calling a received function will not lead to remote invocations.

Cloud Haskell (Epstein et al., 2011) leverages guaranteed-serializable, static closures for a message-passing communication model inspired by Erlang. In contrast, in our model spores are sent between passive, persistent silos. Moreover, the coordination of concurrent activity is based on futures, instead of message passing. Closures and continuations in Termite Scheme (Germain, 2006) are always serializable; references to non-serializable objects (like open files) are automatically wrapped in processes that are serialized as their process ID. Similar to Cloud Haskell, Termite is inspired by Erlang. In contrast to Termite, 
the function passing model is statically typed, enabling advanced type-based optimizations. In non-process-oriented models, parallel closures (Matsakis, 2012) and RiverTrail (Herhut et al., 2013) address important safety issues of closures in a concurrent setting. However, RiverTrail currently does not support capturing variables in closures, which is critical for the apply combinator in the function passing model. In contrast to parallel closures, spores do not require a type system extension in Scala.

Acute ML (Sewell et al., 2005) is a dialect of ML which proposes numerous primitives for distributed programming, such as type-safe serialization, dynamic linking and rebinding, and versioning. The function passing model, in contrast, is based on spores, which ship with their serialized environment or they fail to compile, obviating the need for dynamic rebinding. HashCaml (Billings et al., 2006) is a practical evolution of Acute ML's ideas in the form of an extension to the OCaml bytecode compiler, which focuses on type-safe serialization and providing globally meaningful type names. In contrast, function passing is merely a programming model, which does not require extensions to the Scala compiler.

ML5 (Murphy VII et al., 2007) provides mobile closures verified not to use resources not present on machines where they are applied. This property is enforced transitively (for all values reachable from captured values), which is stronger than what plain spores provide. However, type constraints allow spores to require properties not limited to mobility. Transitive properties are supported either using type constraints based on type classes which enforce a transitive property or by integrating with type systems that enforce transitive properties. Unlike ML5, spores do not require a type system extension. Further, the function passing model sits on top of these primitives to provide a full programming model for distribution, which also integrates spores and type-safe pickling.

MapReduce (Dean \& Ghemawat, 2008), Dryad (Isard et al., 2007), and Apache Spark (Zaharia et al., 2010) are distributed systems for large-scale data processing, building on concepts from functional programming, such as higher-order functions. Ciel (Murray et al., 2011) is an execution engine for distributed data-flow programs which, like the function passing model, supports dynamic task dependencies and data-dependent control flow, thereby going beyond the capabilities of MapReduce and Dryad. Like Spark, MapReduce, and Dryad, Ciel supports transparent scaling, and fault tolerance is transparent. Our system shares several aspects of its design with Spark, such as lazy materialization of datasets, and serialization of computations, including closures, for remote shipping. In contrast to Spark's RDDs (Resilient Distributed Datasets), silos in the function passing model are lower-level abstractions without transparent fault tolerance or transparent scaling. Instead, the goal of our programming model is to provide a foundation on top of which higher-level abstractions for distributed programming, like RDDs, can be built. An important focus of our work is the precise and detailed formalization of lineages and silos as a first step towards formal models of distributed systems like Spark. DryadLINQ (Yu et al., 2008) extends Dryad with a functional language, LINQ, for expressing transformations on distributed datasets which enables sophisticated optimizations including those typically employed by databases. Like the function passing model, DryadLINQ enables the use of function closures within distributed computations. DryadLINQ uses dynamic code generation to ensure the serializability of these closures: captured variables are either eliminated by partial evaluation or serialized as resources shipped to machines in the cluster at runtime. In our system, serializability of closures (spores) is ensured at compile time using macros instead of using dynamic code 
generation. Like Spark, but unlike DryadLINQ and MapReduce, silos may be persisted in memory across multiple usages in our system. Nectar (Gunda et al., 2010) provides a caching service to improve the resource utilization of Dryad/DryadLINQ clusters. For this, Nectar identifies derived datasets by the computations that generate them, similar to lineages in the function passing model. Nectar has been shown to significantly improve space utilization as well as provide speed-ups via incremental computation and shared sub-computations. It would be interesting to investigate potential usages of the lineages of the functional passing model for similar purposes. In contrast to these above systems, the function passing model is meant to act as more of a middleware to facilitate the design and implementation of such systems, and as a result provides finer-grained control over details such as fault handling. Rather than system building and experimental evaluation, our focus is on a precise formalization of the programming model, as well as the proof of preservation and progress properties.

The Clojure programming language proposes agents (Hickey, 2008)-stationary mutable data containers that users apply functions to in order to update an agent's state. The function passing model, in contrast, proposes that data in stationary containers be immutable, and that transformations by function application form a persistent data structure. Further, Clojure's agents are designed to manage state in a shared memory scenario, whereas the function passing model is designed with remote references for a distributed scenario.

The function passing model is also related to the actor model of concurrency (Agha, 1986), which features multiple implementations in Scala (Haller \& Odersky, 2009; Typesafe, 2015; He et al., 2014). Actors can serve as in-memory data containers in a distributed system, like our silos. Unlike silos, actors encapsulate behavior in addition to immutable or mutable values. While only some actor implementations support mobile actors (none in Scala), mobile behavior in the form of serializable closures is central to the function passing model.

\section{Future Work and Conclusion}

\subsection{Ongoing and Future Work}

Our ongoing efforts are three-fold; (a) we are working on a semantics and implementation of fault handling on top of the function passing model, (b) we are exploring approaches for memory reclamation, and (c) we are working to better understand the concerns of separate compilation.

\subsubsection{Fault Handling}

The current implementation of the function passing model includes overloaded variants of function passing's primitive operations to enable flexible fault handling semantics. The main idea is to specify fault handlers for subgraphs of computation DAGs. Our guiding principle is to make the definition of the failure-free path through a computation DAG as simple as possible, while still enabling the handling of faults at the fine-granular level of individual silo references.

What follows are illustrations of this ongoing work based on the running example introduced in Section 2.3. 
Defining fault handlers. Fault handlers may be specified whenever the lineage of a silo reference is extended. For this purpose, the introduced apply primitive is overloaded. For example, consider the running example illustrated in Figure 5, but extended with a fault handler:

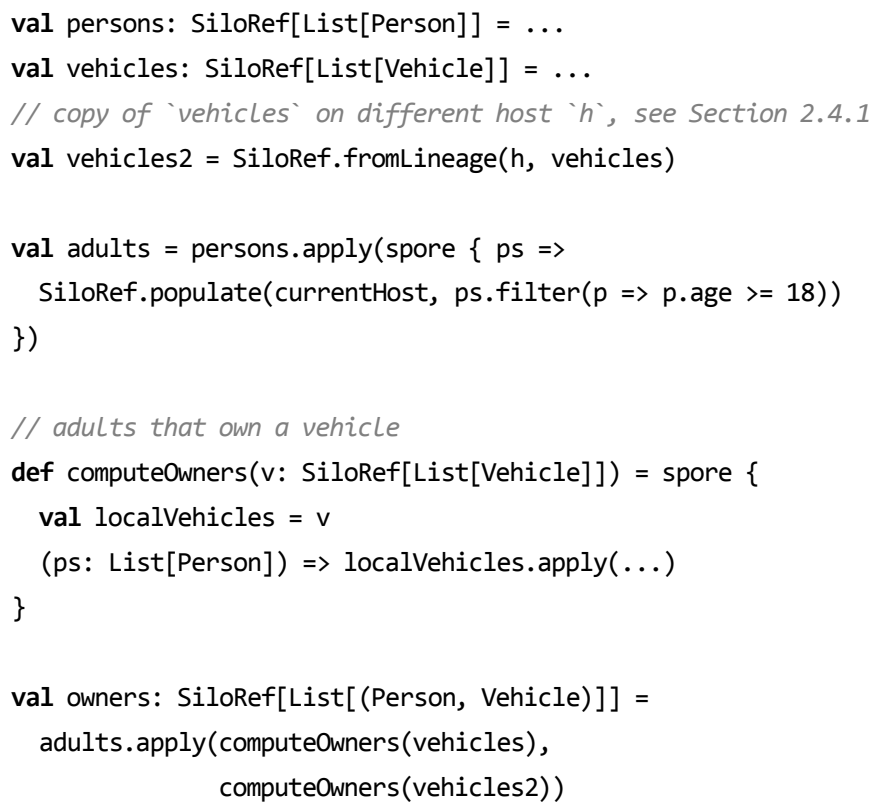

Importantly, in the apply call on the last line, in addition to computeowners (vehicles), the regular spore argument of apply, computeowners (vehicles2) is passed as an additional argument. The second argument registers a failure handler for the subgraph of the computation DAG starting at adults. This means that if during the execution of computeowners (vehicles) it is detected that the vehicles silo reference has failed, it is checked whether the siloRef that the higher-order combinator was invoked on (in this case, adults) has a failure handler registered. In that case, the failure handler is used as an alternative spore to compute the result of adults.apply (..). In this example, we specified computeowners (vehicles2) as the failure handler; thus, in case vehicles has failed, the computation is retried using vehicles 2 instead.

A limitation of this basic failure handling model is the fact that in the above example, the fall-back silo vehicles2 is defined up front using a specific host $\mathrm{h}$. However, note that the computation DAG defined by a SiloRef can easily be materialized on any host using the SiloRef.fromLineage function shown in Section 2.4.1. Thus, assuming the existence of a function that randomly returns one of the healthy hosts in the cluster, say, getHealthyHost(), the above fault handler could be made more dynamic as follows:

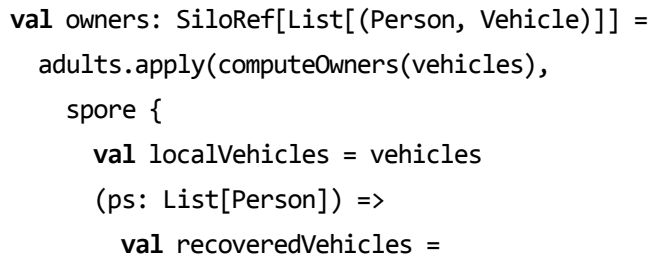




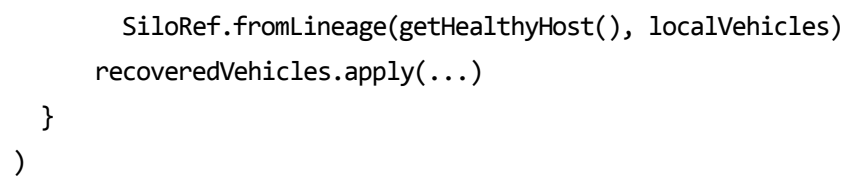

In order to implement more flexible fault handling mechanisms, including strategies for straggler mitigation, additional information pertaining to the execution of (parts of) DAGs would need to be provided. For example, to mitigate stragglers, materializations could be initiated on alternative machines after a timeout. The specification (on the API level) and implementation of more flexible execution policies is left for future work.

\subsubsection{Memory Reclamation}

We are exploring multiple approaches for memory reclamation. The first approach uses Java's weak references to detect when a SiloRef is no longer reachable from local GC roots. Upon detection the host of the corresponding silo is notified to decrease the silo's reference count; the host's reference(s) to the silo are nulled out when the reference count reaches zero. It is important to note that this strategy requires notifying a silo's host whenever a SiloRef to the silo reaches a new machine, to increase the silo's reference count. The second approach leverages uniqueness types in Scala (Haller \& Odersky, 2010; Haller \& Loiko, 2016). Here, SiloRefs are locally unique, and the programmer can explicitly declare a SiloRef as unused; the type system ensures that such an "unused" SiloRef is not used again subsequently. As in the first approach, upon marking a SiloRef as unused, the corresponding silo's host is notified to decrease the silo's reference count.

Other future work includes better understanding concerns of separate compilation in order to evaluate whether our model could be of help in coordinating between microservices. ${ }^{13}$

\subsection{Conclusion}

We have presented the function passing model, a new programming model and new substrate or middleware upon which to build data-centric distributed systems. This enables two important benefits for distributed system builders; since (a) all computations are functional transformations on immutable data, the model directly provides lineages which can form the basis for fault recovery, and (b) communication is made well-typed by design, the function passing model attempts to more naturally model the paradigm of data-centric programming by extending monadic programming to the network. One insight of our model is that lineage-based fault recovery mechanisms, used in widespread frameworks for distribution, are closely related to persistent data structures in functional programming. Therefore, we believe that fault tolerance based on lineages may benefit from further study by the functional programming community. We have also presented a formalization of the function

13 Microservices are small, independent (separately-compiled) services running on different machines which communicate with each other to together make up a single and complex application. They are a predominant trend in industry amongst rich and complicated web-based services. 
passing model, providing an operational semantics and a type system for lineage-based distributed computation. While our formal model does not yet provide an approach to fault tolerance, our hope is that aspects of the model including lineages, silo references, and silo materialization can eventually form the basis of a complete formal treatment of lineagebased fault tolerance in future work. Finally, we have implemented our approach in and for Scala, and have shown that it is possible to support different popular patterns of distributed processing, such as batch processing with Apache Spark's RDDs and MBrace's cloud-based asynchronous tasks. 


\section{A Illustrated Listings}

\section{A.1 Matching Vehicles and Owners}

Figure A 1 shows an illustrated version of the listing in Figure 4.

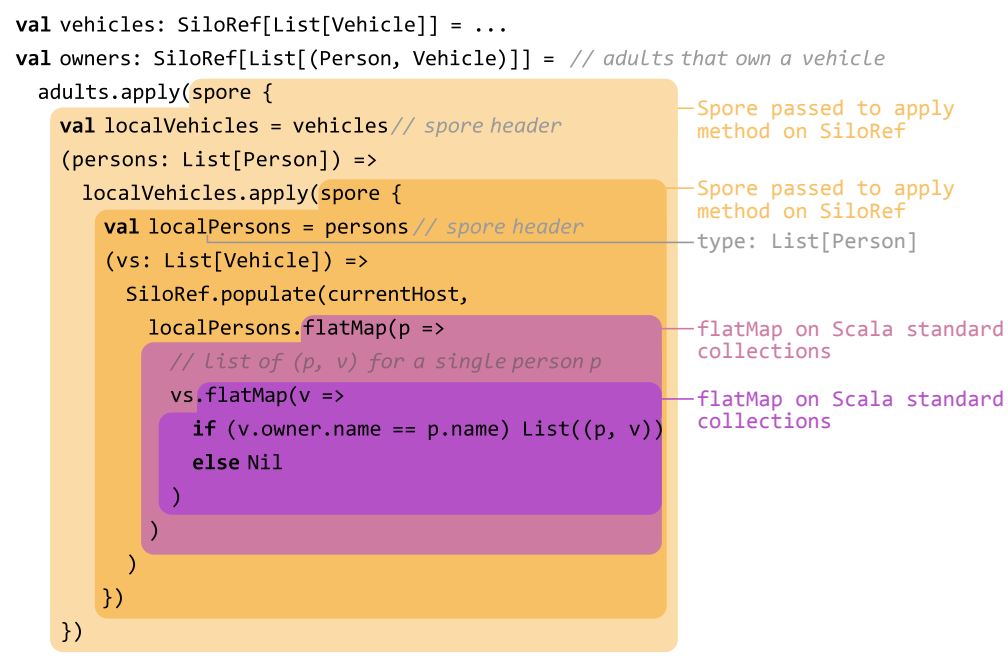

Fig. A 1. Matching persons and vehicle owners using the apply combinator.

\section{A.2 K-Means Clustering}

Figure A 2 shows an illustrated version of the listing of the k-means clustering example in Section 3.3. 


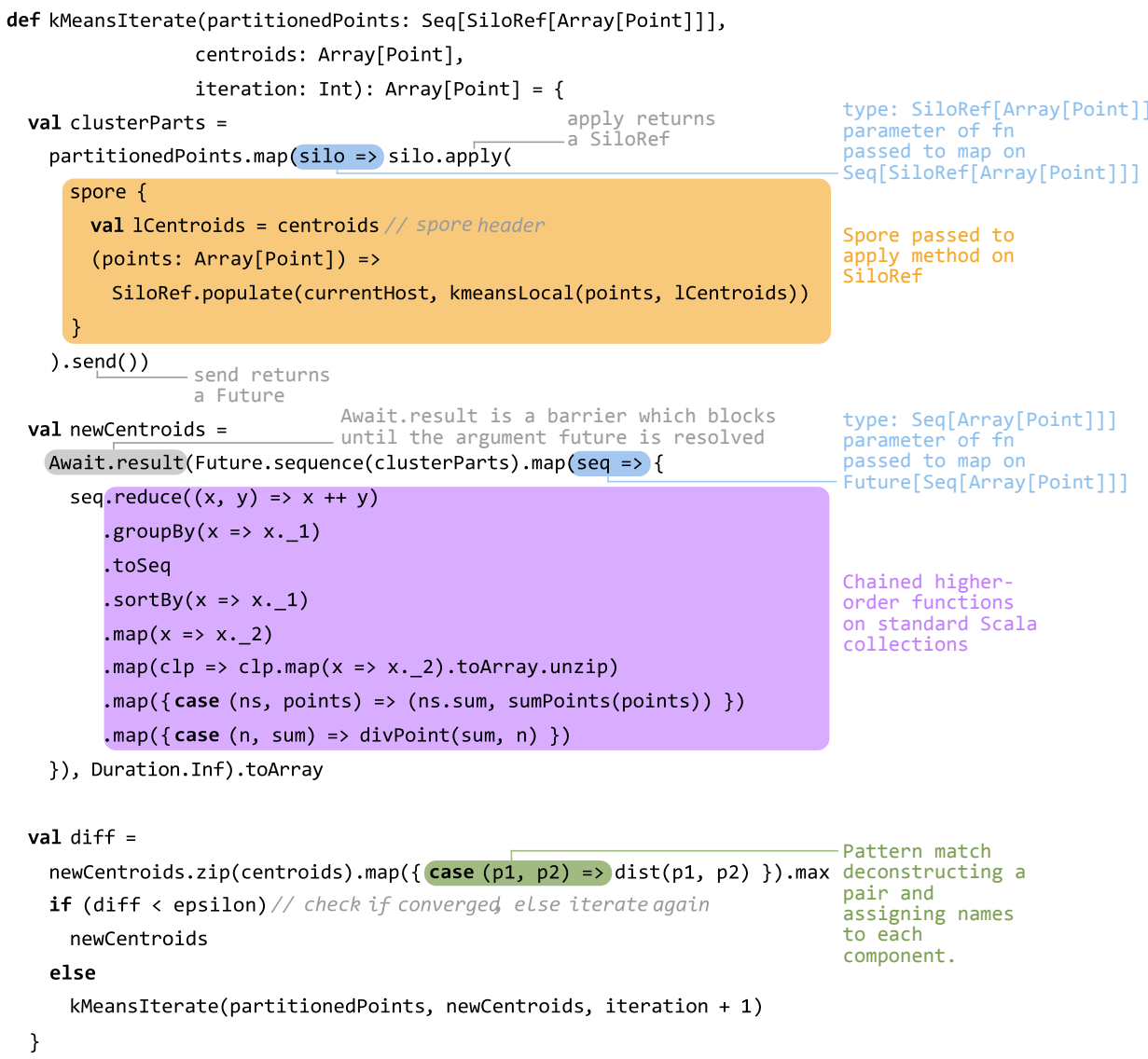

Fig. A 2. Excerpt of an implementation of k-means clustering.

\section{B Proofs}

\section{B.1 Proof of Theorem 4.1}

Theorem (Serializable Values) If $\Gamma ; \Sigma \vdash v: T$ and serializable $(T)$ then $\emptyset ; \Sigma \vdash v: T$.

Proof

By induction on the derivation of $\Gamma ; \Sigma \vdash v: T$ with a case analysis of the last applied rule.

- Cases T-Int, T-Unit, and T-Host are trivial.

- Case T-SiloRef.

1. By the assumptions
(a) $\Gamma ; \Sigma \vdash v: T$
(b) $\operatorname{serializable}(T)$

2. By 1.a) and T-SiloReF
(a) $v=\operatorname{Ref}(l, h)$
(b) $T=\operatorname{SiloRef}\left[T^{\prime}\right]$
(c) $\Sigma($ id $(l))=T^{\prime}$ 
(d) $\Sigma \vdash \operatorname{Ref}(l, h)$

3. By 2.a-d), and T-SiloRef, $\emptyset ; \Sigma \vdash v: T$.

- Case T-Spore follows by S-Spore and the IH.

\section{B.2 Proof of Theorem 5.2}

Lemma B.1 (Weakening) If $\Gamma ; \Sigma \vdash t: T$ and $x \notin \operatorname{dom}(\Gamma)$, then $\Gamma, x: T^{\prime} ; \Sigma \vdash t: T$.

Proof

By induction on the derivation of $\Gamma ; \Sigma \vdash t: T$.

Lemma B.2 (Weakening of Store Typing)

1. If $\Gamma ; \Sigma \vdash t: T$ and $\imath \notin \operatorname{dom}(\Sigma)$ then $\Gamma ; \Sigma^{\prime} \vdash t: T$ where $\Sigma^{\prime}=\left[\imath \mapsto T^{\prime}\right] \Sigma$.

2. If $\Sigma \vdash(t, \sigma)^{h}$ and $\boldsymbol{\imath} \notin \operatorname{dom}(\Sigma)$ then $\Sigma^{\prime} \vdash(t, \sigma)^{h}$ where $\Sigma^{\prime}=[\imath \mapsto T] \Sigma$.

3. If $\Sigma \vdash H$ and $\imath \notin \operatorname{dom}(\Sigma)$ then $\Sigma^{\prime} \vdash H$ where $\Sigma^{\prime}=[\imath \mapsto T] \Sigma$.

Proof

Part 1: By induction on the derivation of $\Gamma ; \Sigma \vdash t: T$. Part 2: By induction on the derivation of $\Sigma \vdash(t, \sigma)^{h}$. Part 3: By induction on the derivation of $\Sigma \vdash H$.

Lemma B.3 (Process) If $\Sigma \vdash \sigma, \Sigma \vdash m$, and process $(h, m, \sigma)=\left(t, M, \sigma^{\prime}\right)$ then $\emptyset ; \Sigma^{\prime} \vdash t: T$ for some $T, \Sigma^{\prime} \vdash M$, and $\Sigma^{\prime} \vdash \sigma^{\prime}$ for some $\Sigma^{\prime} \supseteq \Sigma$.

Proof

- Case Proc-ReQ.

1. By the assumptions
(a) $\Sigma \vdash \sigma$
(b) $\Sigma \vdash m$
(c) $\operatorname{process}(h, m, \sigma)=\left(t, M, \sigma^{\prime}\right)$

2. By Proc-ReQ
(a) $m=\operatorname{Req}\left(h^{\prime}, r, l\right)$
(b) $r=\operatorname{Ref}(l, h)$
(c) $\sigma(i d(l))=(v, P)$
(d) $M=\left\{h^{\prime} \leftarrow \operatorname{Res}(l, v)\right\}$
(e) $\sigma^{\prime}=$ consume $(i d(l), P, \sigma)$
(f) $t=$ unit

3. Define $\Sigma^{\prime}:=\Sigma$.

4. By 2.e) and Def. 4.6, $\operatorname{dom}\left(\sigma^{\prime}\right) \subseteq \operatorname{dom}(\sigma)$.

5. By 1.a), 3., 4., and WF-Store1-3, $\Sigma^{\prime} \vdash \sigma^{\prime}$.

6. By 1.b), 2.a,b), and WF-REQ

(a) $\Sigma(i d(l))=\Sigma(l)$

(b) $\Sigma \vdash r$ 
7. Define $T:=\Sigma(i d(l))$.

8. By 1.a), 2.c), 7., and WF-Store2, $\emptyset ; \Sigma \vdash v: T$.

9. By 6.a), 7., 8., and WF-Res, $\Sigma \vdash \operatorname{Res}(l, v)$.

10. By 2.d), 9., and WF-Messages, $\Sigma \vdash M$.

11. By 2.f), T-Unit, and Lemma B.2, $\emptyset ; \Sigma \vdash t: T^{\prime}$ for some $T^{\prime}$.

12. 3., 5., 10., and 11. close this case.

- Cases Proc-ReqMat1, Proc-ReqMat2, and Proc-ReqParent follow analogously.

- Case Proc-ReqApply.

1. By the assumptions
(a) $\Sigma \vdash \sigma$
(b) $\Sigma \vdash m$
(c) $\operatorname{process}(h, m, \sigma)=\left(t, M, \sigma^{\prime}\right)$

2. By Proc-ReqApply
(a) $m=\operatorname{Req}\left(h^{\prime}, r, \imath\right)$
(b) $r=\operatorname{Ref}(l, h)$
(c) $l=\operatorname{Applied}\left(l^{\prime}, l^{\prime}, p\right)$
(d) $\sigma\left(i d\left(l^{\prime}\right)\right)=(v, P)$
(e) $t=\operatorname{respond}\left(h^{\prime}, l\right.$, await $\left.(\operatorname{send}(p v))\right)$
(f) $\sigma^{\prime}=$ consume $\left(i d\left(l^{\prime}\right), P, \sigma\right)$
(g) $M=\emptyset$

3. By 1.b), 2.a,b), and WF-REQ
(a) $\Sigma(i d(l))=\Sigma(l)$
(b) $\Sigma \vdash r$

4. By 2.b,c), 3.b), WF-ReF, and WF-Lin2
(a) $\Sigma\left(\imath^{\prime}\right)=T$
(b) $\Sigma\left(i d\left(l^{\prime}\right)\right)=T^{\prime}$
(c) $\exists \Gamma . \Gamma ; \Sigma \vdash p: T^{\prime} \Rightarrow$ SiloRef $[T]\{\ldots\}$
(d) $\Sigma \vdash l^{\prime}$

5. By 1.a), 2.d), 4.b), and WF-Store2, $\emptyset ; \Sigma \vdash v: T^{\prime}$.

6. By 4.c), T-SPORE, Def. serializable, and Lemma 4.1, $\emptyset ; \Sigma \vdash p: T^{\prime} \Rightarrow \operatorname{SiloRef}[T]\{\ldots\}$.

7. By 5., 6., and T-AppSpore, $\emptyset, \Sigma \vdash p v:$ SiloRef $[T]$.

8. By 7. and T-SEND, $\emptyset, \Sigma \vdash \operatorname{send}(p v):$ Future $[T]$.

9. By 8. and T-Await, $\emptyset, \Sigma \vdash$ await $(\operatorname{send}(p v)): T$.

10. By 3.a), 4.a), and Def. $4.2, \Sigma(\imath)=T$ and thus by T-IDENT, $\emptyset ; \Sigma \vdash \imath:$ Future $[T]$.

11. By 2.e), 9., 10., and T-Respond, $\emptyset, \Sigma \vdash t$ : Unit.

12. By 2.g) and WF-Messages-Emp, $\Sigma \vdash M$.

13. By 2.f) and Def. 4.6, $\operatorname{dom}\left(\sigma^{\prime}\right) \subseteq \operatorname{dom}(\sigma)$.

14. By 1.a), 13., and WF-STore1-3, $\Sigma \vdash \sigma^{\prime}$.

15. 11., 12., and 14. close this case. 
- Cases Proc-ReqPersist and Proc-Res follow analogously.

\section{Theorem (Subject Reduction)}

1. If $\Gamma ; \Sigma \vdash t: T, \Sigma \vdash \sigma$, and $t\left|\sigma \rightarrow^{h} t^{\prime}\right| \sigma^{\prime}$ then $\Gamma ; \Sigma^{\prime} \vdash t^{\prime}: T$ and $\Sigma^{\prime} \vdash \sigma^{\prime}$ for some $\Sigma^{\prime} \supseteq \Sigma$

2. If $\Sigma \vdash H \mid M$ and $H\left|M \rightarrow H^{\prime}\right| M^{\prime}$ then $\Sigma^{\prime} \vdash H^{\prime} \mid M^{\prime}$ for some $\Sigma^{\prime} \supseteq \Sigma$.

Proof

Part 1: by induction on the derivation of $t\left|\sigma \rightarrow^{h} t^{\prime}\right| \sigma^{\prime}$ with case analysis of the last applied rule.

- Case R-AppAbs.

1. By the assumptions
(a) $\Gamma ; \Sigma \vdash t: T$
(b) $\Sigma \vdash \sigma$
(c) $t\left|\sigma \rightarrow^{h} t^{\prime}\right| \sigma^{\prime}$

2. By R-AppAbs
(a) $t=E\left[\left(\left(x: T^{\prime}\right) \Rightarrow t^{\prime \prime}\right) v^{\prime}\right]$
(b) $t^{\prime}=E\left[\left[x \mapsto v^{\prime}\right] t^{\prime \prime}\right]$
(c) $\sigma^{\prime}=\sigma$

3. By 1.a) and 2.a), $\Gamma ; \Sigma \vdash\left(\left(x: T^{\prime}\right) \Rightarrow t^{\prime \prime}\right) v^{\prime}: T^{\prime \prime}$.

4. By 3. and T-ApP,
(a) $\Gamma ; \Sigma \vdash\left(\left(x: T^{\prime}\right) \Rightarrow t^{\prime \prime}\right): T^{\prime} \Rightarrow T^{\prime \prime}$
(b) $\Gamma ; \Sigma \vdash v^{\prime}: T^{\prime}$

5. By 4.a) and T-ABs, $\Gamma, x: T^{\prime} ; \Sigma \vdash t^{\prime \prime}: T^{\prime \prime}$.

6. By 4.b), 5., and Lemma 5.1, $\Gamma ; \Sigma \vdash\left[x \mapsto v^{\prime}\right] t^{\prime \prime}: T^{\prime \prime}$.

7. By 1.a), 2.a-b), 3., and 6., $\Gamma ; \Sigma \vdash t^{\prime}: T$.

8. 2.c) and 7. close this case.

- Cases R-IntOp, R-AppSpore, and R-Await follow analogously.

- Case R-Apply.

1. By the assumptions
(a) $\Gamma ; \Sigma \vdash t: T$
(b) $\Sigma \vdash \sigma$
(c) $t\left|\sigma \rightarrow^{h} t^{\prime}\right| \sigma^{\prime}$

2. By R-Apply
(a) $t=E[\operatorname{apply}(r, p)]$
(b) $r=\operatorname{Ref}\left(l, h^{\prime}\right)$
(c) $t^{\prime}=E\left[r^{\prime}\right]$
(d) $r^{\prime}=\operatorname{Ref}\left(l^{\prime}, h^{\prime}\right)$
(e) $l^{\prime}=\operatorname{Applied}((h, i), l, p)$ where $i$ fresh 
(f) $\sigma^{\prime}=\sigma$

3. By 1.a) and 2.a), $\Gamma ; \Sigma \vdash \operatorname{apply}(r, p): \hat{T}$.

4. By 3. and T-Apply,
(a) $\hat{T}=\operatorname{siloRef}\left[T^{\prime}\right]$
(b) $\Gamma ; \Sigma \vdash r: \operatorname{SiloRef}\left[T^{\prime \prime}\right]$
(c) $\Gamma ; \Sigma \vdash p: T^{\prime \prime} \Rightarrow$ SiloRef $\left[T^{\prime}\right]\{$ type $\mathscr{C}=\bar{T}\}$

5. By 2.b), 4.b), T-SiLoRef, and WF-ReF
(a) $\Sigma(i d(l))=T^{\prime \prime}$
(b) $\Sigma \vdash r$
(c) $\Sigma \vdash l$
(d) $h^{\prime} \in \mathscr{H}$

6. Define $\Sigma^{\prime}:=\left[(h, i) \mapsto T^{\prime}\right] \Sigma$.

7. By 2.d-e), 4.c), 5.a-d), 6., WF-Lin2, and WF-ReF, $\Sigma^{\prime} \vdash r^{\prime}$.

8. By 2.d-e), 6., 7., and T-SiloRef, $\Gamma ; \Sigma^{\prime} \vdash r^{\prime}: \operatorname{SiloRef}\left[T^{\prime}\right]$.

9. By 2.e), 3., 4.a), 6., and part 1 of Lemma B.2, $\Gamma ; \Sigma^{\prime} \vdash \operatorname{apply}(r, p): \operatorname{SiloRef}\left[T^{\prime}\right]$.

10. By 1.a), 2.e), 6., and part 1 of Lemma B.2, $\Gamma ; \Sigma^{\prime} \vdash t: T$.

11. By 2.a,c), 8., 9., and 10., $\Gamma ; \Sigma^{\prime} \vdash t^{\prime}: T$.

12. By 1.b) and 2.f), $\Sigma \vdash \sigma^{\prime}$.

13. By $6 ., \Sigma^{\prime} \supseteq \Sigma$.

14. By 12., 13., and WF-Store3, $\Sigma^{\prime} \vdash \sigma^{\prime}$.

15. 11., 13., and 14. close this case.

- Cases R-Persist and R-UnPersist follow analogously.

Part 2: by induction on the derivation of $H\left|M \rightarrow H^{\prime}\right| M^{\prime}$ with case analysis of the last applied rule.

- Case R-Local.

1. By the assumptions
(a) $\Sigma \vdash H \mid M$
(b) $H\left|M \rightarrow H^{\prime}\right| M^{\prime}$

2. By R-LocaL
(a) $H=\left\{(t, \sigma)^{h}\right\} \cup H^{\prime \prime}$
(b) $H^{\prime}=\left\{\left(t^{\prime}, \sigma^{\prime}\right)^{h}\right\} \cup H^{\prime \prime}$
(c) $t\left|\sigma \rightarrow^{h} t^{\prime}\right| \sigma^{\prime}$
(d) $M^{\prime}=M$

3. By 1.a) and WF-Config, $\Sigma \vdash H$.

4. By 2.a), 3., and WF-Host2
(a) $\Sigma \vdash(t, \sigma)^{h}$
(b) $\Sigma \vdash H^{\prime \prime}$

5. By 4.a) and WF-HostConfig
(a) $\Sigma \vdash \sigma$ 
(b) $\Gamma ; \Sigma \vdash t: T$ for some $\Gamma$

6. By 2.c), 5.a,b), and part 1

(a) $\Gamma ; \Sigma^{\prime} \vdash t^{\prime}: T$

(b) $\Sigma^{\prime} \vdash \sigma^{\prime}$ for some $\Sigma^{\prime} \supseteq \Sigma$

7. By $6 . \mathrm{a}, \mathrm{b})$ and WF-HostConfig, $\Sigma^{\prime} \vdash\left(t^{\prime}, \sigma^{\prime}\right)^{h}$.

8. By 4.b), 6.b), and part 3 of Lemma B.2, $\Sigma^{\prime} \vdash H^{\prime \prime}$.

9. By 2.b), 7., 8., and WF-Нолт2, $\Sigma^{\prime} \vdash H^{\prime}$.

10. By 1.a), 2.d), 9., WF-Config, and WF-Messages, $\Sigma^{\prime} \vdash H^{\prime} \mid M^{\prime}$.

- Case R-Send.

1. By the assumptions
(a) $\Sigma \vdash H \mid M$
(b) $H\left|M \rightarrow H^{\prime}\right| M^{\prime}$

2. By R-SEND
(a) $H=\left\{(E[\operatorname{send}(r)], \sigma)^{h}\right\} \cup H^{\prime \prime}$
(b) $H^{\prime}=\left\{(E[i d(l)], \sigma)^{h}\right\} \cup H^{\prime \prime}$
(c) $r=\operatorname{Ref}\left(l, h^{\prime}\right)$
(d) $m=\operatorname{Req}(h, r, i d(l))$
(e) $M^{\prime}=M \uplus\left\{h^{\prime} \leftarrow m\right\}$

3. By 1.a) and WF-Config
(a) $\Sigma \vdash H$
(b) $\Sigma \vdash M$

4. By 2.a), 3.a), and WF-Host2
(a) $\Sigma \vdash(E[\operatorname{send}(r)], \sigma)^{h}$
(b) $\Sigma \vdash H^{\prime \prime}$

5. By 4.a) and WF-HostConfig
(a) $\Sigma \vdash \sigma$
(b) $\Gamma ; \Sigma \vdash E[\operatorname{send}(r)]: T$ for some $\Gamma$

6. By 5.b), $\Gamma ; \Sigma \vdash \operatorname{send}(r): \hat{T}$.

7. By 6 . and T-SEND
(a) $\hat{T}=$ Future $\left[T^{\prime \prime}\right]$
(b) $\Gamma ; \Sigma \vdash r: \operatorname{SiloRef}\left[T^{\prime \prime}\right]$

8. By 2.c), 7.b), and T-SiLoReF
(a) $\Sigma(i d(l))=T^{\prime \prime}$
(b) $\Sigma \vdash r$

9. By 8.a) and T-IDENT, $\Gamma ; \Sigma \vdash i d(l)$ : Future $\left[T^{\prime \prime}\right]$.

10. By 5.b), 6., 7.a), and 9., $\Gamma ; \Sigma \vdash E[i d(l)]: T$.

11. By 5.a), 10., and WF-HostConfig, $\Sigma \vdash(E[i d(l)], \sigma)^{h}$.

12. By 4.b), 11., and WF-Host2, $\Sigma \vdash H^{\prime}$. 
13. By 2.c,d), 8.b), and WF-ReQ, $\Sigma \vdash m$.

14. By 2.c,e), 3.b), 8.b), 13., WF-Ref, and WF-Messages, $\Sigma \vdash M^{\prime}$.

15. By 12., 14., and WF-Config, $\Sigma \vdash H^{\prime} \mid M^{\prime}$.

- Cases R-Populate and R-Respond follow analogously.

- Case R-Process.

1. By the assumptions

(a) $\Sigma \vdash H \mid M$

(b) $H\left|M \rightarrow H^{\prime}\right| M^{\prime}$

2. By R-Process
(a) $H=\left\{(E[\text { await }(l)], \sigma)^{h}\right\} \cup H^{\prime \prime}$
(b) $H^{\prime}=\left\{\left(E[t \text {; await }(\imath)], \sigma^{\prime}\right)^{h}\right\} \cup H^{\prime \prime}$
(c) $M^{\prime}=\hat{M} \uplus M^{\prime \prime}$
(d) $\operatorname{process}(h, m, \sigma)=\left(t, M^{\prime \prime}, \sigma^{\prime}\right)$
(e) $M=\hat{M} \uplus\{h \leftarrow m\}$

3. By 1.a) and WF-Config
(a) $\Sigma \vdash H$
(b) $\Sigma \vdash M$

4. By 2.a), 3.a), and WF-Host2
(a) $\Sigma \vdash(E[\text { await }(\imath)], \sigma)^{h}$
(b) $\Sigma \vdash H^{\prime \prime}$

5. By 4.a) and WF-HostConfig
(a) $\Sigma \vdash \sigma$
(b) $\Gamma ; \Sigma \vdash E[$ await $(l)]: T$ for some $\Gamma$

6. By 2.e), 3.b), and WF-Messages, $\Sigma \vdash m$.

7. By 2.d), 5.a), 6., and Lemma B.3 (Process), $\exists \Sigma^{\prime}$ such that
(a) $\emptyset ; \Sigma^{\prime} \vdash t: T^{\prime}$
(b) $\Sigma^{\prime} \vdash M^{\prime \prime}$
(c) $\Sigma^{\prime} \vdash \sigma^{\prime}$
(d) $\Sigma^{\prime} \supseteq \Sigma$

8. By 5.b), 7.d), and part 1 of Lemma B.2, $\Gamma ; \Sigma^{\prime} \vdash E[\operatorname{await}(l)]: T$.

9. By 7.a) and $8 ., \Gamma ; \Sigma^{\prime} \vdash E[t$; await $(l)]: T$.

10. By 7.c), 9., and WF-HostConfig, $\Sigma^{\prime} \vdash\left(E[t \text {; await }(l)], \sigma^{\prime}\right)^{h}$.

11. By 4.b), 7.d), and part 3 of Lemma B.2, $\Sigma^{\prime} \vdash H^{\prime \prime}$.

12. By 2.b), 10., 11., and WF-Host2, $\Sigma^{\prime} \vdash H^{\prime}$.

13. By 3.b), 7.d), WF-Res, WF-ReQ, WF-ReF, and WF-Messages, $\Sigma^{\prime} \vdash M$.

14. By 2.c), 7.b), 13., and WF-Messages, $\Sigma^{\prime} \vdash M^{\prime}$.

15. By 12., 14., and WF-Config, $\Sigma^{\prime} \vdash H^{\prime} \mid M^{\prime}$.

- Case R-Process-Val follows analogously. 


\section{B.3 Proof of Theorem 5.5}

Lemma B.4 (Responsive Population) Let $\Sigma \vdash H|M| R$ and $H=\left\{(E[t], \sigma)^{h}\right\} \uplus \hat{H}$.

Then $\forall h^{\prime} \in \operatorname{hosts}(H):\left\{(E[\operatorname{send}(r)], \sigma)^{h}\right\} \uplus \hat{H}\left|M \uplus\left\{h^{\prime} \leftarrow m\right\} \rightarrow^{*} H^{\prime}\right| M^{\prime} \uplus\{h \leftarrow m\}$ after a finite number of reduction steps where $r=\operatorname{Ref}\left(l, h^{\prime}\right), l=\operatorname{Mat}(l)$ for some $\boldsymbol{l}$, and $m=\operatorname{Res}(\boldsymbol{l}, v)$.

\section{Proof Sketch}

By R-Send, $\left\{(E[\operatorname{send}(r)], \sigma)^{h}\right\} \uplus \hat{H}\left|M \uplus\left\{h^{\prime} \leftarrow m\right\} \rightarrow\left\{(E[\imath], \sigma)^{h}\right\} \uplus \hat{H}\right| M \uplus\left\{h^{\prime} \leftarrow m\right\} \uplus$ $\left\{h^{\prime} \leftarrow m^{\prime}\right\}$ where $m^{\prime}=\operatorname{Req}(h, r, \imath)$. By Def. 5.1 message $m$ is processed by $h^{\prime}$ after a finite number of reduction steps. As a result, the store of $h^{\prime}$ is updated with the mapping $l \mapsto v$. After another finite number of reduction steps, $h^{\prime}$ processes message $m^{\prime}$. By R-Process, R-Process-Val, and Proc-ReQMat1, the resulting multiset of messages includes $\{h \leftarrow$ $\operatorname{Res}(l, v)\}$ as required.

\section{Lemma B.5 (Responsive Apply)}

Proof

1. By R-Local and R-Apply

(a) $E[\operatorname{apply}(r, p)]\left|\sigma \rightarrow^{h} E\left[r^{\prime}\right]\right| \sigma \mid\left\{r^{\prime}\right\}$

(b) $r^{\prime}=\operatorname{Ref}\left(l^{\prime}, h^{\prime}\right)$

(c) $r=\operatorname{Ref}\left(l, h^{\prime}\right)$

(d) $l^{\prime}=\operatorname{Applied}((h, i), l, p)$ where $i$ fresh

(e) $H^{\prime}=\left\{\left(E\left[r^{\prime}\right], \sigma\right)^{h}\right\} \uplus \hat{H}$

(f) $M^{\prime}=M$

(g) $R^{\prime}=R \uplus\left\{r^{\prime}\right\}$

2. By Lemma 5.4, Responsive $\left(H^{\prime}, M^{\prime}, R\right)$.

3. Consider $H^{\prime}\left|M^{\prime} \uplus\left\{h^{\prime} \leftarrow m\right\}\right| R^{\prime}$ where $m=\operatorname{Req}\left(h^{\prime \prime}, r^{\prime}, \iota\right)$.

4. By Def. $5.1 H^{\prime}\left|M^{\prime} \uplus\left\{h^{\prime} \leftarrow m\right\}\right| R^{\prime} \rightarrow^{*} H_{p}\left|M_{p}\right| R_{p}$ such that $\left(E_{p}\left[\operatorname{await}\left(l_{p}\right)\right], \sigma_{p}\right)^{h^{\prime}} \in$ $H_{p} \vee\left(E_{p}\left[v_{p}\right], \sigma_{p}\right)^{h^{\prime}} \in H_{p}$ and $\left\{h^{\prime} \leftarrow m\right\} \in M_{p}$.

5. There are two cases. Case 1: $i d(l) \notin \operatorname{dom}\left(\sigma^{\prime}\right)$. In this case $H_{p}\left|M_{p}\right| R_{p}$ can be reduced according to R-Process (or R-Process-VAL) and Proc-ReQParent. As a result, $h^{\prime}$ reduces send $\left(\operatorname{Ref}\left(l, h^{\prime}\right)\right)$. By R-SEND, $\operatorname{Ref}\left(l, h^{\prime}\right) \in R$, and Lemma 5.4, $H_{p}\left|M_{p}\right| R_{p} \rightarrow^{*}$ $H_{r}\left|M_{r} \uplus\left\{h^{\prime} \leftarrow \operatorname{Res}(i d(l), v)\right\}\right| R_{r}$ after a finite number of reduction steps. By Def. 5.1 and Proc-Res, $H_{r}\left|M_{r} \uplus\left\{h^{\prime} \leftarrow \operatorname{Res}(i d(l), v)\right\}\right| R_{r} \rightarrow^{*} H^{\prime \prime}\left|M^{\prime \prime}\right| R^{\prime \prime}$ after a finite number of reduction steps, such that

(a) $H^{\prime \prime}=\left\{\left(E^{\prime}\left[t^{\prime}\right], \sigma^{\prime}\right)^{h^{\prime}}\right\} \cup H_{3}$ where $t^{\prime}=v^{\prime}$ or $t^{\prime}=\operatorname{await}\left(\imath^{\prime}\right)$

(b) $M^{\prime \prime}=\left\{h^{\prime} \leftarrow m\right\} \uplus M_{3}$

(c) $\sigma^{\prime}(i d(l))=v$

(d) $\Sigma^{\prime \prime} \vdash H^{\prime \prime}\left|M^{\prime \prime}\right| R^{\prime \prime}$

Case 2: $\sigma^{\prime}(i d(l))=v$. In this case $H^{\prime \prime}=H_{p}, M^{\prime \prime}=M_{p}$, and $R^{\prime \prime}=R_{p}$.

6. By 5.a-c), R-Process, R-Process-VAL, and Proc-ReQApPLY

(a) $\operatorname{process}\left(h^{\prime}, m, \sigma^{\prime}\right)=\left(t^{\prime \prime}, \emptyset, \sigma^{\prime}\right)$

(b) $t^{\prime \prime}=\operatorname{respond}\left(h^{\prime \prime}, l\right.$, await $\left.(\operatorname{send}(p v))\right)$

(c) $H^{\prime \prime}\left|M^{\prime \prime}\right| R^{\prime \prime} \rightarrow H_{3}\left|M_{3}\right| R^{\prime \prime}$

(d) $H_{3}=\left\{\left(E^{\prime}\left[t^{\prime \prime} ; t^{\prime}\right], \sigma^{\prime}\right)^{h^{\prime}}\right\} \cup H_{4}$ 
7. By 5.d), 6.c), and Theorem 5.2 (Subject Reduction), $\Sigma_{3} \vdash\left\{\left(E^{\prime}\left[t^{\prime \prime} ; t^{\prime}\right], \sigma^{\prime}\right)^{h^{\prime}}\right\} \cup H_{4}\left|M_{3}\right| R^{\prime \prime}$ for some $\Sigma_{3} \supseteq \Sigma^{\prime \prime}$.

8. By 7., WF-ConfIG, WF-Host2, and WF-HostConfig

(a) $\Sigma_{3} \vdash \sigma^{\prime}$

(b) $\Gamma_{3} ; \Sigma_{3} \vdash E^{\prime}\left[t^{\prime \prime} ; t^{\prime}\right]: T_{3}$ for some $\Gamma_{3}, T_{3}$

9. By $6 . b), 8 . b)$, and the type rules

(a) $\Gamma_{4} ; \Sigma_{3} \vdash p: T^{\prime} \Rightarrow \operatorname{SiloRef}[T]\{$ type $\mathscr{C}=\bar{T}\}$ for some $\Gamma_{4}$

(b) $p=$ spore $\left\{\overline{x: T=v} ;\left(x: T^{\prime}\right) \Rightarrow t\right\}$

10. By 9.a,b), and T-SPORE

(a) $\overline{x: T}, x: T^{\prime} ; \emptyset \vdash t:$ SiloRef $[T]$

(b) $\forall S \in \bar{T}$, SiloRef $[T]$. serializable $(S)$

11. By the type rules, derivation 10.a) does not contain applications of T-SiLoREF or TIDENT. By 10.b) spore $p$ does not capture futures. Thus, any occurrence of await $(\hat{\imath})$ within $p v$ is preceded by a reduction of a corresponding send resulting in future $\hat{\imath}$. By Lemma 5.4 (Responsiveness), Responsive $\left(H_{3}, M_{3}, R\right)$. There are two cases.

Case 1: $d=0$. Then $p$ does not contain a nested apply invocation. Therefore, by Lemma B.4, $p v$ reduces to a value $r^{\prime \prime}$ after a finite number of reduction steps. Since either $r^{\prime \prime} \in R$ or $r^{\prime \prime}$ is newly populated, send $\left(r^{\prime \prime}\right)$ results in a response $h^{\prime} \leftarrow \operatorname{Res}\left(i d\left(r^{\prime \prime}\right), v^{\prime \prime}\right)$ after a finite number of reduction steps. This enables $h^{\prime}$ to reduce respond $\left(h^{\prime \prime}, l, v^{\prime \prime}\right)$ which concludes this case.

Case 2: $d>0$. The depth of nested applys of term $p v$ is less than the depth of the term apply $(r, p)$. Therefore, by the induction hypothesis, reductions of nested apply invocations within $p v$ result in responsive configurations. By Lemma B.4, $p v$ reduces to a value $r^{\prime \prime}$ after a finite number of reduction steps. Since either $r^{\prime \prime} \in R$, or $r^{\prime \prime}$ is newly populated, or $r^{\prime \prime}$ is the result of a nested apply invocation, send $\left(r^{\prime \prime}\right)$ results in a response $h^{\prime} \leftarrow \operatorname{Res}\left(i d\left(r^{\prime \prime}\right), v^{\prime \prime}\right)$ after a finite number of reduction steps. This enables $h^{\prime}$ to reduce respond $\left(h^{\prime \prime}, \boldsymbol{l}, v^{\prime \prime}\right)$ which concludes this case.

Theorem (Finite Materialization) Let $\Sigma \vdash H|M| R$ such that Responsive $(H, M, R)$. If $H|M| R \rightarrow H^{\prime}\left|M^{\prime}\right| R^{\prime}$ then Responsive $\left(H^{\prime}, M^{\prime}, R^{\prime}\right)$.

Proof

Corollary of Lemma 5.4, Lemma B.4, and Lemma B.5.

\section{References}

Agha, G. (1986). ACTORS: A model of concurrent computation in distributed systems. MIT Press.

Agha, Gul A., Mason, Ian A., Smith, Scott F., \& Talcott, Carolyn L. (1997). A foundation for actor computation. Journal of functional programming, 7(1), 1-72.

Apache. (2015). Hadoop. http://hadoop. apache.org/.

Billings, John, Sewell, Peter, Shinwell, Mark, \& Strniša, Rok. (2006). Type-safe distributed programming for OCaml. Pages 20-31 of: Proceedings of the 2006 workshop on ML. ACM.

Chambers, Craig, Raniwala, Ashish, Perry, Frances, Adams, Stephen, Henry, Robert R., Bradshaw, Robert, \& Weizenbaum, Nathan. (2010). FlumeJava: Easy, efficient data-parallel pipelines. Pages 363-375 of: PLDI. 
Dean, Jeffrey, \& Ghemawat, Sanjay. (2008). MapReduce: Simplified data processing on large clusters. Commun. ACM, 51(1), 107-113.

Dzik, Jan, Palladinos, Nick, Rontogiannis, Konstantinos, Tsarpalis, Eirik, \& Vathis, Nikolaos. (2013). MBrace: Cloud computing with monads. Pages 7:1-7:6 of: 0001, Tim Harris, \& Madhavapeddy, Anil(eds),PLOS@SOSP.ACM.

Elsman, Martin. (2005). Type-specialized serialization with sharing. Pages 47-62 of: Trends in Functional Programming.

Epstein, Jeff, Black, Andrew P., \& Jones, Simon L. Peyton. (2011). Towards Haskell in the cloud. Pages 118-129 of: Haskell Symposium.

Germain, Guillaume. (2006). Concurrency oriented programming in Termite Scheme. Page 20 of: Erlang.

Gunda, Pradeep Kumar, Ravindranath, Lenin, Thekkath, Chandramohan A., Yu, Yuan, \& Zhuang, Li. (2010). Nectar: Automatic management of data and computation in datacenters. Pages 75-88 of: Arpaci-Dusseau, Remzi H., \& Chen, Brad (eds), OSDI. USENIX Association.

Haller, Philipp, \& Loiko, Alex. (2016). LaCasa: Lightweight affinity and object capabilities in Scala. Pages 272-291 of: Visser, Eelco, \& Smaragdakis, Yannis (eds), OOPSLA. ACM.

Haller, Philipp, \& Odersky, Martin. (2009). Scala actors: Unifying thread-based and event-based programming. Theoretical computer science, 410(2), 202-220.

Haller, Philipp, \& Odersky, Martin. (2010). Capabilities for uniqueness and borrowing. Pages 354378 of: ECOOP 2010, Maribor, Slovenia, June 21-25, 2010.

Haller, Philipp, Prokopec, Aleksandar, Miller, Heather, Klang, Viktor, Kuhn, Roland, \& Jovanovic, Vojin. (2012). Futures and promises. http://docs.scala-Lang.org/overviews/core/futures.html.

He, Jiansen, Wadler, Philip, \& Trinder, Philip. (2014). Typecasting actors: From Akka to TAkka. Pages 23-33 of: Scala.

Herhut, Stephan, Hudson, Richard L., Shpeisman, Tatiana, \& Sreeram, Jaswanth. (2013). River trail: A path to parallelism in javascript. Pages 729-744 of: OOPSLA.

Hickey, Rich. (2008). The Clojure programming language. Page 1 of: DLS. ACM.

Isard, Michael, Budiu, Mihai, Yu, Yuan, Birrell, Andrew, \& Fetterly, Dennis. (2007). Dryad: Distributed data-parallel programs from sequential building blocks. Pages 59-72 of: EuroSys.

Kennedy, Andrew. (2004). Pickler combinators. J. funct. program., 14(6), 727-739.

Matsakis, Nicholas D. (2012). Parallel closures: A new twist on an old idea. HotPar.

Miller, Heather, Haller, Philipp, Burmako, Eugene, \& Odersky, Martin. (2013). Instant pickles: Generating object-oriented pickler combinators for fast and extensible serialization. Pages 183202 of: OOPSLA.

Miller, Heather, Haller, Philipp, \& Odersky, Martin. (2014). Spores: A type-based foundation for closures in the age of concurrency and distribution. Pages 308-333 of: ECOOP.

Milner, R., Parrow, J., \& Walker, D. (1992). A calculus of mobile processes. Information and computation, 100(1), 1-77.

Murphy VII, Tom, Crary, Karl, \& Harper, Robert. (2007). Type-safe distributed programming with ML5. Pages 108-123 of: TGC.

Murray, Derek Gordon, Schwarzkopf, Malte, Smowton, Christopher, Smith, Steven, Madhavapeddy, Anil, \& Hand, Steven. (2011). CIEL: A universal execution engine for distributed data-flow computing. Andersen, David G., \& Ratnasamy, Sylvia (eds), NSDI. USENIX Association.

NICTA. (2015). Scoobi. https://github.com/nicta/scoobi.

Odersky, Martin, \& Zenger, Matthias. (2005). Scalable component abstractions. Pages 41-57 of: Johnson, Ralph E., \& Gabriel, Richard P. (eds), OOPSLA. ACM.

Odersky, Martin, Spoon, Lex, \& Venners, Bill. (2010). Programming in Scala. Second edn. Artima. 
Peyton Jones, Simon, Gordon, Andrew, \& Finne, Sigbjorn. (1996). Concurrent Haskell. Pages 295308 of: POPL.

Pierce, Benjamin C. (2002). Types and programming languages. MIT Press.

Rossberg, Andreas, Le Botlan, Didier, Tack, Guido, Brunklaus, Thorsten, \& Smolka, Gert. (2004). Alice through the looking glass. Trends in functional programming, 5, 79-96.

Sewell, Peter, Leifer, James J, Wansbrough, Keith, Nardelli, Francesco Zappa, Allen-Williams, Mair, Habouzit, Pierre, \& Vafeiadis, Viktor. (2005). Acute: High-level programming language design for distributed computation. Pages 15-26 of: ACM SIGPLAN Notices, vol. 40. ACM.

Shapiro, Marc, Preguiça, Nuno M., Baquero, Carlos, \& Zawirski, Marek. (2011). Conflict-free replicated data types. Pages 386-400 of: Défago, Xavier, Petit, Franck, \& Villain, Vincent (eds), SSS. Lecture Notes in Computer Science, vol. 6976. Springer.

Twitter. (2015). Scalding. https://github.com/twitter/scalding.

Typesafe. (2015). Akka. http://akka.io/.

Waldo, Jim, Wyant, Geoff, Wollrath, Ann, \& Kendall, Samuel C. (1996). A note on distributed computing. Pages 49-64 of: MOS.

Yu, Yuan, Isard, Michael, Fetterly, Dennis, Budiu, Mihai, Erlingsson, Úlfar, Gunda, Pradeep Kumar, \& Currey, Jon. (2008). DryadLINQ: A system for general-purpose distributed data-parallel computing using a high-level language. Pages 1-14 of: Draves, Richard, \& van Renesse, Robbert (eds), OSDI. USENIX Association.

Zaharia, Matei, Chowdhury, Mosharaf, Franklin, Michael J., Shenker, Scott, \& Stoica, Ion. (2010). Spark: Cluster computing with working sets. Pages 10-10 of: Proceedings of the 2Nd USENIX Conference on Hot Topics in Cloud Computing. HotCloud'10. Berkeley, CA, USA: USENIX Association. 